\title{
Structure of lipid bilayers
}

\author{
John F. Nagle ${ }^{a, b, *}$, Stephanie Tristram-Nagle ${ }^{b}$ \\ a Department of Physics, Carnegie Mellon University, Pittsburgh, PA 15213, USA \\ b Department of Biological Sciences, Carnegie Mellon University, Pittsburgh, PA 15213, USA
}

Received 10 May 2000; received in revised form 22 August 2000; accepted 22 August 2000

\begin{abstract}
The quantitative experimental uncertainty in the structure of fully hydrated, biologically relevant, fluid $\left(L_{\alpha}\right)$ phase lipid bilayers has been too large to provide a firm base for applications or for comparison with simulations. Many structural methods are reviewed including modern liquid crystallography of lipid bilayers that deals with the fully developed undulation fluctuations that occur in the $L_{\alpha}$ phase. These fluctuations degrade the higher order diffraction data in a way that, if unrecognized, leads to erroneous conclusions regarding bilayer structure. Diffraction measurements at high instrumental resolution provide a measure of these fluctuations. In addition to providing better structural determination, this opens a new window on interactions between bilayers, so the experimental determination of interbilayer interaction parameters is reviewed briefly. We introduce a new structural correction based on fluctuations that has not been included in any previous studies. Updated measurements, such as for the area compressibility modulus, are used to provide adjustments to many of the literature values of structural quantities. Since the gel $\left(L_{\beta}{ }^{\prime}\right)$ phase is valuable as a stepping stone for obtaining fluid phase results, a brief review is given of the lower temperature phases. The uncertainty in structural results for lipid bilayers is being reduced and best current values are provided for bilayers of five lipids. (C) 2000 Elsevier Science B.V. All rights reserved.
\end{abstract}

Keywords: Lipid bilayer; X-ray diffraction; Structure determination; Fluctuation; Hydration; Interaction

\section{Introduction}

This is a review of the venerable, but still active, topic of lipid bilayer structure. Lipid bilayer structural data are used for a variety of purposes in biophysics, such as consideration of hydrophobic matching of intrinsic membrane proteins. We shall not attempt to review all the applications, but will concentrate instead on providing reliable data for general use. This project deserves considerable discussion and analysis. However, the user in a hurry

\footnotetext{
* Corresponding author. Fax: +1-412-681-0648;

E-mail: nagle@andrew.cmu.edu
}

can find our current bottom-line values in Table 6 in Section 12 as well as comparison values in Tables 3 and 5 .

This review is closest in content to the influential BBA review of Rand and Parsegian published over 10 years ago [1]. Although that review emphasized bilayer interactions, extensive tables of structural data for many bilayers were given. In comparison, the present review includes fewer lipid bilayers. We emphasize and compare the different results obtained by different methods for some of the most popular lipids, DPPC, DMPC, DOPC, EPC and DLPE.

Much of the difficulty in obtaining good quantitative structure for the biologically relevant, fully hydrated, fluid $\left(L_{\alpha}\right)$ phase is due to the intrinsic pres- 
ence of fluctuations. A related topic is the interactions between bilayers. Interactions are connected with structure determination because interactions are present in the most useful, multilamellar vesicle (MLV), samples which are used to determine structure. On the other hand, quantitative structure is a precursor to quantitative evaluation of interactions. Structure determination and interactions are also connected because fluctuations play a central role in both. However, to avoid undue length, this review will focus primarily on structure.

This review focuses on experimental methods for obtaining bilayer structure. An alternative is computer simulations. This alternative is becoming increasingly attractive with the rapid progress in simulations because the level of detail is so much greater than can be obtained experimentally. This detail can even be a guide to the interpretation of experimental results [2,3]. Of course, simulations are no better than the models (force fields) that are simulated, and sometimes worse because of limitations to small systems and short times. Reliable experimental data, though incomplete, provides a guide to modeling and a necessary check on the reliability of simulations.

At this point some readers may challenge our assertion that lipid bilayer structure should still be considered an active area. It has a long and rich history. Many prominent biophysicists have published in it and moved on. Users of bilayer structural data have many references to choose from and each user has a favorite. Such a reader should examine Fig. 1 which shows literature values for a particularly central quantity, namely, the average interfacial area $A$ per lipid molecule for DPPC bilayers at $50^{\circ} \mathrm{C}$ in the biologically relevant, fully hydrated, fluid ( $F$, synon-

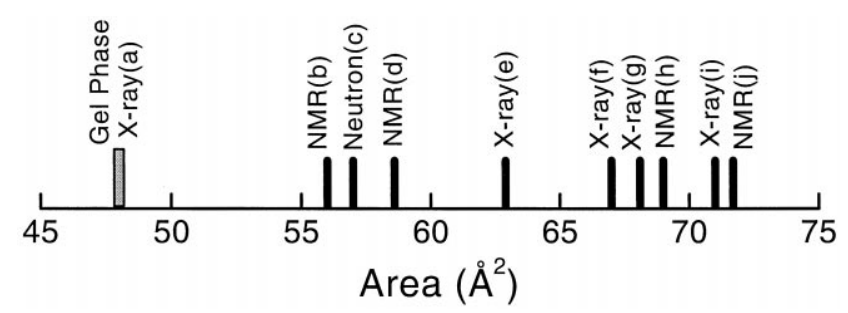

Fig. 1. Summary of published areas for fluid phase DPPC at $50^{\circ} \mathrm{C}$ (black) and gel phase DPPC (grey) at $20^{\circ} \mathrm{C}$. References: ${ }^{\mathrm{a}}$ Sun et al. [4], ${ }^{\mathrm{b}}$ Pace and Chan [9], ${ }^{\mathrm{c}}$ Büldt et al. [10], ${ }^{\mathrm{d}}$ Schindler and Seelig [11], ${ }^{\mathrm{e}}$ Nagle et al. [3], ${ }^{\mathrm{f}}$ Lewis and Engelman [12], ${ }^{\mathrm{g}}$ Rand and Parsegian [1] and Janiak et al. [13], ${ }^{\mathrm{h}}$ DeYoung and Dill [14], ${ }^{\mathrm{i}}$ Lis et al. [15], ${ }^{\mathrm{j}}$ Thurmond et al. [16]. ymously, the $L_{\alpha}$ or liquid crystalline) phase. Such scatter cannot be attributed to sample difference since DPPC has been synthesized to high purity for 25 years. The scatter in these $A_{\mathrm{DPPC}}^{\mathrm{F}}$ values, all for the same state of the same lipid, is unacceptably large for guiding computer simulations, which are sensitive to differences of about $1 \AA^{2}$. The scatter in $A_{\mathrm{DPPC}}^{\mathrm{F}}$ is even larger when viewed from the perspective of comparing to the gel $(\mathrm{G})$ phase, for which $A_{\mathrm{DPPC}}^{\mathrm{G}}=47.9 \AA^{2}$ [4]. The difference, $A_{\mathrm{DPPC}}^{\mathrm{F}}-A_{\mathrm{DPPC}}^{\mathrm{G}}$, measures the effect of fluidization which is what makes the bilayer biologically relevant. If one employs the intellectually impoverished method of obtaining a value by uncritically averaging all literature values, one would still face an uncertainty in $A_{\mathrm{DPPC}}^{\mathrm{F}}-A_{\mathrm{DPPC}}^{\mathrm{G}}$ at the $50 \%$ level. The mean thickness of the bilayer is also inversely proportional to $A$ and is therefore subject to comparable scatter that degrades important quantitative discussions of hydrophobic matching [5-8]. This review will hopefully convince the reader that structural quantities are no longer so poorly determined as indicated by Fig. 1 . This will involve a critical review of many of the methods that gave those results. In addition, in Section 7, we introduce a new correction based on fluctuations that has not been included in any of the previous analyses, including our own; using this correction we provide adjustments to literature values of $A$. We also use new values of material moduli [17] to revise some of the earlier structural values given by ourselves and by Rand and Parsegian [1]. Although everyone agrees that the fluid $L_{\alpha}$ phase is the most important one for biology, the so-called gel $\left(L_{\beta}{ }^{\prime}\right)$ phase is valuable as a stepping stone for obtaining fluid phase results, so results for other, more ordered, lamellar phases are briefly reviewed in Section 11. In Section 6 a brief survey is given of recent work on the effects of fluctuations on the determination of interbilayer interactions. First, we turn in the next section to what one can hope to achieve for the structure of lipid bilayers and we define some of the terms that are used.

\section{What is meant by lipid bilayer structure?}

It is often supposed that determining bilayer structure by diffraction means doing crystallography. 


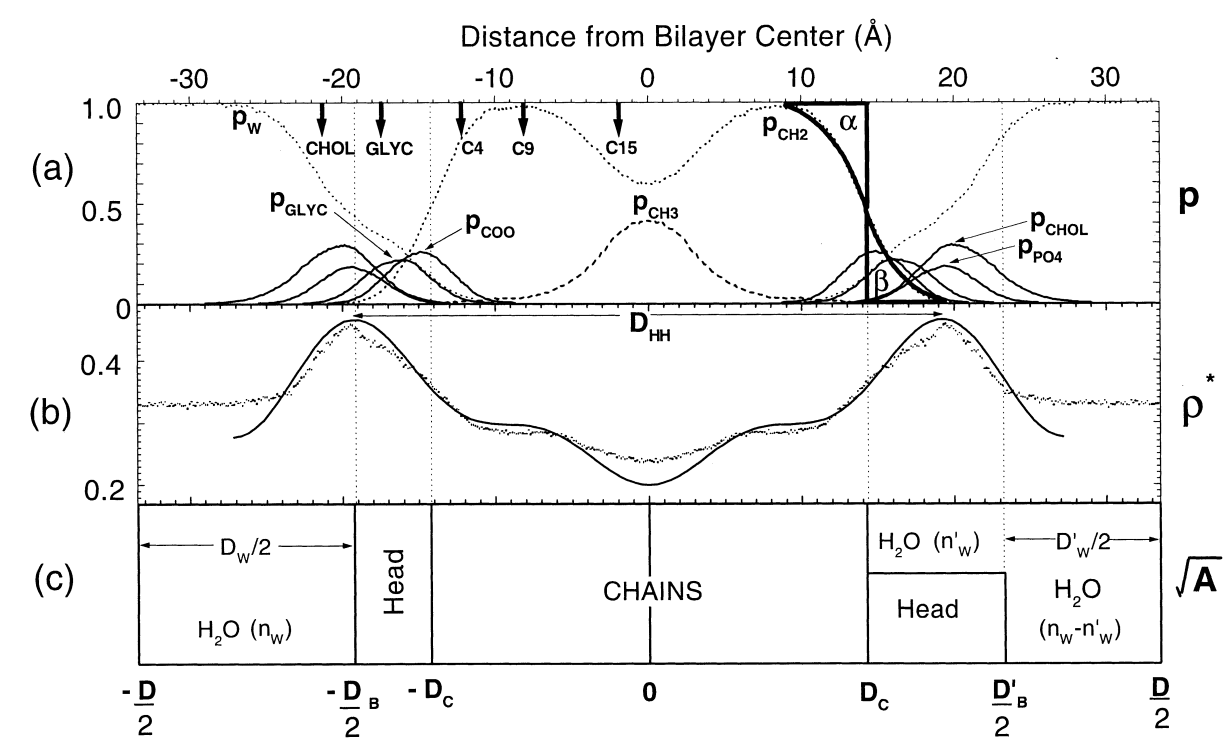

Fig. 2. Three representations of structure of DPPC bilayers in the $L_{\alpha}$ fluid phase. (a) Probability distribution functions $p$ for different component groups from simulations [20] and the downward pointing arrows show the peak locations determined by neutron diffraction with $25 \%$ water [10]. The equality of the areas denoted $\alpha$ and $\beta$ locates the Gibbs dividing surface for the hydrocarbon region determined by the simulation. (b) Electron density profile $\rho^{*}$ from X-ray studies (solid line) [3] and from simulations (dots) (contributed by Scott Feller). (c) Two volumetric pictures. The version on the left monolayer is a simple three compartment representation. The version on the right monolayer is a more realistic representation of the interfacial headgroup region [26]. $D_{\mathrm{C}}$ is the experimentally determined Gibbs dividing surface for the hydrocarbon region. The $x$-axis is in $\AA$ along the bilayer normal with the same scale for $\mathrm{a}, \mathrm{b}$ and $\mathrm{c}$. The $y$-axis in $\mathrm{c}$ shows a lateral dimension along the surface of the bilayer. Values for the parameters in $\mathrm{c}$ are taken from Table 6.

While lipid crystallography has been pursued and is illuminating [18], it is important to recognize that fully hydrated lipid bilayers are not even close to being in a crystalline state. The contrast is strongest for bilayers that are in the fluid, $L_{\alpha}$ phase where the hydrocarbon chains are conformationally disordered in contrast to the nearly all-trans chains in lipid crystals. Even for the conformationally ordered gel and subgel bilayer phases, there are substantial differences compared to the crystal structures. These differences are not surprising since there is much more water in fully hydrated lipid bilayers, which substantially alters the balance of interaction energies of the bilayers compared to the nearly dry crystalline state and which also allows for increased fluctuations. Because of the fluctuations, it makes no sense to contemplate an atomic level structure for biologically relevant lipid bilayers [19]. The absence of such structures should not be blamed on poor diffraction technique or on sample preparation; rather, such structures simply do not exist in the biologically relevant state.
The appropriate description for the positions of atoms in the lipid molecule is that of broad statistical distribution functions. Fig. 2a shows simulations for distribution functions for the component groups of DPPC along the direction of the bilayer normal [20]. Most users of such information focus on the peak positions of the distributions. Equally important are the shapes of the distributions. At first glance, one would simply describe the shapes by their widths; in Fig. 2a the full widths at half maximum are of order $5 \AA$. However, one should also realize that such distributions are only Gaussians if the potential of mean force happens to be harmonic, and this would be strictly accidental. Non-Gaussian and skewed distributions occur most certainly for the terminal methyl distribution for methyls limited to lipids in one monolayer [21-23] (the distribution in Fig. 2a is automatically symmetric because it includes methyls from both monolayers). Skewness warns one that the average position of a component group is not necessarily the position of the maximum in the distribution. Of course, if one is trying to fit 
limited amounts of data, it is convenient to limit the fitting functions to Gaussians that are parameterized just by a mean position and a width. The errors in making this approximation have been assessed and improvements are indicated when the Gaussian assumption is not made, although for volumetric applications the improvements are not large [22]. However, there is a different application, namely, for the positions of methylenes as a function of carbon number, where using the most probable (peak) value in the non-Gaussian distribution gives different values and a different qualitative picture than using true averages. Using averages shows that the mean distance between successive methylenes decreases towards the methyl end [2]; this is consistent with the usual picture of increasing disorder towards the bilayer center. In contrast, using peak values in the distribution suggests wrongly that the successive distances are nearly constant (we are indebted to R.G. Snyder for bringing this example to our attention).

So far, the description has been exclusively along the spatial direction of the bilayer normal. In contrast, in the lateral direction along the bilayer, the distribution functions for the $L_{\alpha}$ phase are just constants because the lipid molecules are in a two-dimensional fluid phase. For the lower temperature phases, however, there is interesting and valuable in-plane structure $[4,24]$ which is reviewed in Section 12.

Fluctuations in fully hydrated fluid phase bilayers mean that X-ray diffraction data from multilamellar arrays of lipid bilayers can only yield electron density profiles (EDP) such as the one shown in Fig. 2b. The peaks in this DPPC electron density profile are associated with the electron dense phosphate group and the lower electron density in the center is associated with the hydrocarbon region and especially with the low electron density of terminal methyl groups of the fatty acids. Therefore, electron density profiles confirm the usual picture of bilayer structure and they give a measure of the bilayer thickness, namely, the head-head separation, $D_{\mathrm{HH}}$. However, electron density profiles only provide a good measure for the location of the phosphate group. Information about the z-coordinates of other groups has been obtained using neutron diffraction, reviewed in Section 8, either with selective deuteration of various component groups (see the arrows in Fig. 2a) ([10], see p. 689), or combined with X-ray diffraction [25].

The transverse description of the bilayer as a set of distribution functions along the $z$-axis is valuable, but it does not include other important information, such as $A$ in the lateral direction, or the volumes of component groups of the lipid molecule. Therefore, a complementary description of bilayer structure is appropriate [26]. The simplest such description, due to Luzzati [27] is shown on the left half of Fig. 2c. For multilamellar arrays with repeat spacing $D$ the volume is divided into two regions. The first region consists of the volume $V_{\mathrm{L}}$ of the lipid and the second region consists of the volume $n_{\mathrm{W}} V_{\mathrm{W}}$ of the water where $V_{\mathrm{W}}$ is the volume of one water molecule. The full thickness of the bilayer region is defined to be $D_{\mathrm{B}}=2 V_{\mathrm{L}} / A$ and the full thickness of the water region is then $D_{\mathrm{W}}=2 n_{\mathrm{W}} V_{\mathrm{W}} / A=D-D_{\mathrm{B}}$.

The volume $V_{\mathrm{L}}$ of the lipid molecule is further divided into two regions, a hydrocarbon chain region and a headgroup region. This division emphasizes another important aspect of bilayer thickness, namely, the thickness $2 D_{\mathrm{C}}$ of the hydrophobic core. We include in $D_{\mathrm{C}}$ all the hydrocarbon chain carbons except for the carbonyl carbon which has substantial hydrophilic character. For DPPC the hydrophobic core therefore consists of 14 methylenes and one terminal methyl on each of the two chains. With this convention the headgroup is then defined to consist of the remainder of the more hydrophilic part of the lipid, which can be subdivided into the carbonyls, glycerol, phosphate and choline. (Another convention is to define the headgroup to be just the phosphate and the choline.) The half thickness of the hydrocarbon region is related to the hydrocarbon volume of the lipid $V_{\mathrm{C}}$ by $D_{\mathrm{C}}=V_{\mathrm{C}} / A$.

In view of the broad distributions shown in Fig. 2a, the boundaries drawn in Fig. 2c are clearly artificially sharp, but Fig. 2c is an appropriate average description in the sense that the sharp lines can be justified as Gibbs dividing surfaces [28]. For example, the $D_{\mathrm{C}}$ line cuts the methylene distribution at a probability near 0.5 in Fig. 2a. (The actual dividing surface criterion is that the integrated probability of methylenes outside $D_{\mathrm{C}}$, indicated by the region marked $\beta$ in Fig. 2 a, should be equal to the integrated deficit probability inside $D_{\mathrm{C}}$, indicated by the region marked $\alpha$.) It may also be noted that, 
even ignoring fluctuations, there are methylenes on the $s n-2$ chain and carbonyls on the $s n-1$ chain that are on the wrong side of $D_{\mathrm{C}}$ because of the inequivalence of the two chains in DPPC; this again is included in the spirit of Gibbs dividing surfaces [28].

To obtain a more realistic picture of the interface region, it is useful to consider a refinement to the simple description on the left side of Fig. 2c. This refinement, shown on the right side of Fig. 2c, explicitly mixes the heads and water in the polar, interfacial region. This gives better correspondence with the simulated distribution functions for the headgroup components in Fig. 2a. In particular, the steric bilayer thickness, defined to be $D_{\mathrm{B}}{ }^{\prime}$, lies in the tails of the distribution function of the choline component in Fig. 2a, whereas the volume delimited by $D_{\mathrm{B}}$ includes less than half of the choline component.

It is appropriate for structural studies to obtain values for all four of these membrane thicknesses $\left(D_{\mathrm{HH}}, D_{\mathrm{B}}, D_{\mathrm{B}}{ }^{\prime}\right.$ and $\left.D_{\mathrm{C}}\right)$ and to determine what relations exist between them. It may be helpful to the reader to note that a glossary of terms along with simple relations between them is included in the Appendix.

\section{Some precise structural quantities}

\subsection{Volumes}

The preceding section emphasizes that volumes are the pivotal quantity to relate lateral structure, such as $A$, to transverse structure, such as the bilayer thickness $D_{\mathrm{B}}$, using relations like $A D_{\mathrm{B}}=2 V_{\mathrm{L}}$. Measurements of total lipid volume $V_{\mathrm{L}}$ have been performed using a variety of techniques. Our favorite method employs neutral flotation in which the density of the aqueous solvent is varied by mixing $\mathrm{D}_{2} \mathrm{O}$ with $\mathrm{H}_{2} \mathrm{O}$, combined with dilatometry which measures volume changes as a function of temperature $[29,30]$. The density of the lipid is then given by the density of the aqueous mixture in which the bilayers neither sink nor float. However, this method is restricted to lipids that have densities intermediate between $\mathrm{D}_{2} \mathrm{O}$ and $\mathrm{H}_{2} \mathrm{O}$. Completely different methods employ a differential vibrating tube densimeter [31,32], differential weighing [33] or buoyant forces
[34]. Values of $V_{\mathrm{L}}$ for different lipids are given in Table 1. Agreement between the different methods is about 3 parts in 1000 and the errors in each method alone is of order 2 parts in 1000 . It may be noted that many papers in the literature have assumed that the partial specific volume of the lipid equals that of water and have simply used $v_{\mathrm{L}}=1 \mathrm{ml} / \mathrm{g}$. As can be seen from Table 1, this is not a bad approximation for many phospholipids in the $L_{\alpha}$ phase, but it is considerably poorer for the gel phase.

The volumes of the chains, $V_{\mathrm{C}}$, and the headgroups, $V_{\mathrm{H}}$, have been obtained for the gel phase of DPPC [4]. As is reviewed in Section 11, the lateral packing dimensions of the all-trans hydrocarbon chains in the gel phase of DPPC can be obtained. Multiplying the lateral area by the longitudinal distance per methylene $(1.27 \AA)$ along the chains gives the volume of the methylenes $V_{\mathrm{CH}_{2}}$. Analysis of the methyl trough in the $\mathrm{X}$-ray low-angle data gave $V_{\mathrm{CH}_{3}}=1.93 V_{\mathrm{CH}_{2}}$ [39]. The reason for the much larger volume of a terminal methyl, despite having only one additional hydrogen, is due to its having an extra hemispherical endcap of steric excluded volume compared to a methylene that is covalently bonded in both directions along the chain. Thence the total hydrocarbon volume, $V_{\mathrm{C}}$, and the headgroup volume $V_{\mathrm{H}}=V_{\mathrm{L}}-V_{\mathrm{C}}$ follow for the gel phase of DPPC. Our best value of $V_{\mathrm{H}}$ is $319 \AA^{3}$ [4], which is quite close to

Table 1

Comparison of literature values for volume/lipid

\begin{tabular}{|c|c|c|c|c|}
\hline Lipid & $\begin{array}{l}\text { Temperature } \\
\left({ }^{\circ} \mathrm{C}\right)\end{array}$ & Ref. & $\begin{array}{l}v_{\mathrm{L}} \\
(\mathrm{ml} / \mathrm{g})\end{array}$ & $\begin{array}{l}V_{\mathrm{L}} \\
\left(\AA^{3} / \text { molecule }\right)\end{array}$ \\
\hline \multirow[t]{4}{*}{ DPPC } & 20 & [30] & 0.939 & 1144 \\
\hline & & [33] & 0.939 & 1144 \\
\hline & & [29] & 0.937 & 1142 \\
\hline & & [35] & 0.940 & 1145 \\
\hline \multirow[t]{5}{*}{ DPPC } & 50 & [30] & 1.011 & 1232 \\
\hline & & [29] & 1.009 & 1230 \\
\hline & & [29] & 1.008 & 1228 \\
\hline & & [35] & 1.009 & 1230 \\
\hline & & [32] & 1.006 & 1226 \\
\hline \multirow[t]{4}{*}{ DMPC } & 30 & [29] & 0.977 & 1100 \\
\hline & & [36] & 0.978 & 1101 \\
\hline & & [35] & 0.978 & 1101 \\
\hline & & [32] & 0.972 & 1094 \\
\hline \multirow[t]{2}{*}{ EPC } & 30 & [36] & 0.988 & 1261 \\
\hline & 20 & [37] & 0.981 & 1252 \\
\hline \multirow[t]{2}{*}{ DOPC } & 30 & [38] & 0.999 & 1303 \\
\hline & 22 & [25] & 0.993 & 1296 \\
\hline
\end{tabular}


the value of $325 \AA^{3}$ suggested by Small [40]. Some earlier values from our lab that were in the range 340-348 $\AA^{3}[29,39,41]$ used less well determined values for the wide-angle packing.

For fluid phases of phosphatidylcholines the volume of the heads has been estimated based on the argument that $V_{\mathrm{H}}$ is the same in the fully hydrated fluid phase as in the fully hydrated gel phase because the headgroup is fully immersed in water in both phases. This assumption also implies that $V_{\mathrm{H}}$ is the same for all lipids with the same PC headgroup. The measured change in lipid volume [29] is then equated to the change in $V_{\mathrm{C}}$. The volume $V_{\mathrm{CH}_{3}}$ of a terminal methyl is often assumed to be about $2 V_{\mathrm{CH}_{2}}$ [29,42], although it was once suggested that a ratio closer to 1.2 applies for the fluid phase [40]. Analysis of combined neutron and X-ray data for fluid phase DOPC obtains a ratio of 2.1 [43] and computer simulations yield a ratio in the range 1.9-2.1 [3] with later simulations favoring 1.9 [22]. Using a ratio near 2 then allows one to estimate the average $V_{\mathrm{CH}_{2}}$ and $V_{\mathrm{CH}_{3}}$ from $V_{\mathrm{C}}$, as shown in Table 2.

Estimates of the volumes of all the component groups on the lipid molecule have been obtained from computer simulations for fluid phase DPPC [20] and fluid phase DOPC and POPC [22]. The method assumes that the average volume of each group is independent of its transverse distance from the center of the bilayer. The resulting volumes must satisfy an independent check that suggests that this assumption is a good approximation. There are only fairly minor variations in the component volumes for the different PC lipids studied and a composite set of

Table 2

Volumes of component groups for general $L_{\alpha}$ phase lecithins ignoring temperature dependence

\begin{tabular}{ll}
\hline Group & Volume $\left(\AA^{3}\right)$ \\
\hline $\mathrm{CH}_{3}$ & $52.7 \pm 1.2^{\mathrm{b}}$ \\
& $53.9 \pm 0.8^{\mathrm{a}}$ \\
$\mathrm{CH}_{2}$ & $28.1 \pm 0.1^{\mathrm{b}}$ \\
& $28.4 \pm 0.4^{\mathrm{a}}$ \\
$\mathrm{HC}=\mathrm{CH}$ & $45.0 \pm 1.6^{\mathrm{b}}$ \\
Carbonyl & $39.0 \pm 1.4^{\mathrm{b}}$ \\
Glycerol & $68.8 \pm 9.9^{\mathrm{b}}$ \\
Phosphate & $53.7 \pm 2.4^{\mathrm{b}}$ \\
Choline & $120.4 \pm 5.0^{\mathrm{b}}$ \\
\hline
\end{tabular}

a This work.

${ }^{\mathrm{b}}$ Armen et al. [22]. volumes, reproduced in Table 2, was given [22]. It is noteworthy that the simulation results in Table 2 give $V_{\mathrm{H}}=321 \AA^{3}$, in very good agreement with the experimental value for the gel phase [4]. The simulations also suggest that the component volumes do not change significantly with hydration level, which is consistent with the experimental result that total lipid volume does not change measurably with hydration [44].

\subsection{Lamellar repeat spacings $D$}

\subsubsection{Accuracy}

Most diffraction studies have been performed on stacks of bilayers, especially on the easily prepared dispersions consisting of multilamellar vesicles (MLVs). The easiest diffraction result to obtain accurately is the repeat spacing $D$, which is always given to at least two significant figures and often to three significant figures, such as $D=67.2 \AA$ for fully hydrated DPPC at $50^{\circ} \mathrm{C}$ [3]. In fact, with the best instrumental resolution $\left(0.0001 \AA^{-1}\right)$ and by fitting line shapes to find the center of the diffraction peak, it is possible to obtain nearly four significant figures, such as $55.06 \AA$ [3]. Such high accuracy is not used in structure determination - two significant figures usually suffice - but it leads into an interesting discussion regarding the nature of the samples.

The MLVs in a random dispersion presumably come in a variety of sizes. Once formed, each bilayer is influenced by its neighbors. It is usually assumed that such MLVs are 'onion-like', consisting of closed concentric spheres, at least in the topological, if not the strict geometric, sense. Since lipid exchange between bilayers and solvent is slow, it is likely that the number of lipids in each bilayer remains constant over fairly long times. The swelling of such MLVs with temperature changes might be expected to be non-uniform depending upon their original degree of flaccidness. There are therefore many reasons to imagine that the $D$ spacing might be different between different MLVs in the same sample, or even within the same MLV - the inner bilayers versus the outer bilayers. It is therefore remarkable that highly precise X-ray diffraction, which detects many MLVs simultaneously, almost always sees lamellar diffraction peaks that are very narrow. If we suppose, for the sake of discussion, that a lamellar peak is a com- 
posite of many peaks, each with a different $D$ spacing, then the observed peak widths would correspond to a distribution of $D$ spacings in the sample with a width less than $\pm 0.05 \AA$. Only once, with a damaged sample, did we observe heterogeneous $D$ spacings. Indeed, there is excellent reason to believe that the observed narrow widths are not even due to polydispersity in the $D$ spacings in the above sense because finite size effects and fluctuations that will be discussed in Section 5 fully account for the shapes of the observed diffraction peaks with a single $D$ [45].

Why then, are diffractionists loathe to quote four significant figures for $D$ ? Although there is at most only one narrow distribution of $D$ values in a single data set, nominally identical samples often have different values of $D$, equally narrowly determined. As usual, the most egregious example is the fully hydrated fluid phase DPPC for which the same study [45] reported four different values of $D$ from $64.5 \AA$ to $67.2 \AA$; other studies reported values ranging down to $60 \AA$ [46]. Another type of variation in $D$ that occurs in a single sample was first noted by Peter Rand (private communication) and confirmed by us. When there is an air bubble in a sample, $D$ becomes smaller as the beam is positioned closer to the bubble. Although all this irreproducibility might appear to be devastating, it is not. Near full hydration the balance of interbilayer forces is rather delicate and the free energy difference caused by variations of a few $\AA$ in $D$ is small $[1,47]$. Basically, all that varies is a small amount of water between the bilayers which does not affect structure and is easily dealt with by considering the continuous Fourier transform of the electron density profile to be discussed in Section 5.

\subsubsection{Oriented samples and the vapor pressure paradox}

Bilayers in MLVs are isotropically oriented in space and therefore give so-called powder patterns (even though they may be thoroughly hydrated). It is convenient that such samples do not have to be (indeed, they cannot be) especially oriented in an Xray beam. Furthermore, there is no concern with mosaic spread that occurs in any real aligned sample and that involves another experimental parameter. However, only a small fraction of the lipid in a powder sample diffracts from a given beam, so intensities are weak. There is also the potential irregularity in the MLVs discussed in the previous paragraph which apparently does not affect $D$ but which certainly reduces the correlation length of the domains within which the sample scatters coherently. For all these reasons it would be valuable to orient the stacks of bilayers. The simplest alignment procedure is to squeeze lipid between two flat substrates, but the strong absorption of X-rays by a substrate has led many researchers to try to orient the lipid on a single substrate and to hydrate the lipid from the vapor [44,48-51]. Another preparation uses free-standing films [52].

An important section of the Rand and Parsegian review [1] concerned the vapor pressure paradox (VPP). The result for all preparations of oriented samples since the 1970s until quite recently was that the measured $D$ was consistently smaller, by more than $5 \AA$, than for fully hydrated samples. Rand and Parsegian [1] noted that a reduction of relative humidity to $99 \%$ would suffice to explain this reduction in $D$, but the experimental care and concern for maintaining the relative humidity of the vapor at $100 \%$ was emphasized $[1,44]$. Since the chemical potential of water is the same for liquid as for saturated vapor, such a reduction in $D$ was inconsistent thermodynamically, so this was aptly named the vapor pressure paradox [1] and it was suggested that there was an intrinsic physical reason for it $[1,44]$. The first paradigm shift regarding the VPP was that it was overcome, though with some effort, for the gel phase of DPPC [41,53]. This suggested that the VPP was associated with the excess fluctuations that occur in the fluid phase and an elegant theory that involved suppression of these fluctuations by the substrate was developed mathematically [55]. We also interpreted some indirect experimental evidence in support of this explanation [54].

Recently, however, Katsaras has reported that there really is no VPP [56]. This breakthrough occurred using neutron diffraction which has the advantage that aluminum is fairly transparent to neutrons, so the sample chamber has no need for special windows upon which vapor can condense as in X-ray chambers. Katsaras produced a massive aluminum sample chamber with excellent temperature and humidity control [57]. Then the fully hydrated $D$ spac- 
ing was obtained in all phases with oriented stacks (a) immersed in water and (b) hydrated from saturated water vapor. Also, under controlled osmotic pressure the $D$ for oriented stacks is the same as for MLVs [58]. This latter paper also showed why this was really consistent with the earlier theory [55]. Katsaras has more recently produced a new sample chamber for X-ray diffraction of oriented samples which the authors have used. We have also used a Peltier cooler in our own chamber to effectively produce fully or even supersaturated vapor. Both methods now give the same $D$ as for MLVs in the $L_{\alpha}$ phase.

The spectre of the VPP has retarded the use of oriented samples for studying fully hydrated lipid bilayers. Now that the VPP has been truly exorcised, oriented samples promise to become more useful for obtaining electron density profiles (see Section 5) because their diffraction signals are so much stronger. However, for obtaining $D$ spacings for fully hydrated samples, it is still more certain to use MLVs. Furthermore, MLVs will likely continue to be the standard sample for obtaining $D$ as a function of osmotic pressure $P$, using the important and convenient method of polymer addition $[1,59]$. By comparison, the conventional X-ray measurement of $P$ for oriented samples hydrated from the vapor using saturated salt solutions are likely to be less reliable near full hydration where $P$ is small. Instead of that conventional method, an alternative has been suggested [58], namely, to use a standard $D$ vs. $P$ curve from
MLVs to obtain $P$ for oriented samples from their measured $D$.

Before concluding this section, there is a potential fallacy regarding whether fully hydrated bilayers are biologically relevant since biological systems exist in salt solutions with relative humidities near $98 \%$. However, the osmotic pressure responsible for changing the structure of bilayers in MLVs is induced by the difference of the relative humidities of the solution outside MLVs and the solution inside MLVs. No such osmotic pressure can be induced on single membranes (except to extract what little water is contained within the membrane). Although the specific effects of binding of ions should not be neglected, especially for charged lipid bilayers, the 'fully hydrated' condition, including solutions which have salt which partitions equally into the water contained in MLVs, is generally the most biologically relevant hydration condition.

\section{Gravimetric X-ray methods}

\subsection{Gravimetric $X$-ray $(G X)$ method}

A conceptually elegant and much used method to obtain $A$, commonly known as the Luzzati method, employs gravimetric, volume and $\mathrm{X}$-ray measurements and is called the GX method in this review. Although the original equations were intuitively rather opaque, the fundamentals are best understood

Table 3

Comparison of literature values for area/lipid ${ }^{\mathrm{a}}$

\begin{tabular}{|c|c|c|c|c|c|}
\hline \multirow[t]{2}{*}{ Method } & \multicolumn{5}{|l|}{ Lipid } \\
\hline & DPPC (gel) & $\operatorname{DPPC}\left(L_{\alpha}\right)$ & DMPC $\left(L_{\alpha}\right)$ & $\operatorname{DOPC}\left(L_{\alpha}\right)$ & $\operatorname{EPC}\left(L_{\alpha}\right)$ \\
\hline \multirow[t]{3}{*}{ GX } & $\mathbf{5 2 . 3}^{1}\left(25^{\circ} \mathrm{C}\right)$ & $71.2^{1}\left(50^{\circ} \mathrm{C}\right)$ & $65.2^{1}\left(27^{\circ} \mathrm{C}\right)$ & $\mathbf{8 2 . 0}^{1}\left(25^{\circ} \mathrm{C}\right)$ & $75.6^{1}\left(25^{\circ} \mathrm{C}\right)$ \\
\hline & $\mathbf{5 0}^{2}\left(21^{\circ} \mathrm{C}\right)$ & $68^{2}\left(50^{\circ} \mathrm{C}\right)$ & $\mathbf{6 2 . 2}^{2}\left(37^{\circ} \mathrm{C}\right)$ & $\mathbf{7 0}^{3}\left(2^{\circ} \mathrm{C}\right)$ & $\mathbf{7 1 .} 7^{4,5}\left(25^{\circ} \mathrm{C}\right)$ \\
\hline & $\mathbf{4 8 . 6}^{6}(\mathrm{RT})$ & - & - & - & $\mathbf{6 4}^{7}$ (RT) \\
\hline \multirow[t]{2}{*}{ GXC } & $48.6^{8}\left(25^{\circ} \mathrm{C}\right)$ & $\mathbf{6 8 . 1}^{8}\left(50^{\circ} \mathrm{C}\right)$ & $\mathbf{6 1 . 7 ^ { 8 }}\left(27^{\circ} \mathrm{C}\right)$ & $\mathbf{7 2 . 1}^{8}\left(25^{\circ} \mathrm{C}\right)$ & $69.5^{8}\left(25^{\circ} \mathrm{C}\right)$ \\
\hline & - & - & $\mathbf{5 9 . 5}^{9}\left(30^{\circ} \mathrm{C}\right)$ & - & - \\
\hline EDP & $47.9^{10}\left(20^{\circ} \mathrm{C}\right)$ & $62.9^{11}\left(50^{\circ} \mathrm{C}\right)$ & $59.7^{12}\left(30^{\circ} \mathrm{C}\right)$ & $72.2^{13}\left(30^{\circ} \mathrm{C}\right)$ & $69.4^{12}\left(30^{\circ} \mathrm{C}\right)$ \\
\hline Neutron & - & $57^{14}\left(50^{\circ} \mathrm{C}\right)$ & - & $\mathbf{5 9 . 3}^{15}(\mathrm{RT})$ & - \\
\hline Unilamellar & - & $66.5^{16}\left(44^{\circ} \mathrm{C}\right)$ & $65.7^{16}\left(36^{\circ} \mathrm{C}\right)$ & $\mathbf{7 0 . 1}^{16}\left(20^{\circ} \mathrm{C}\right)$ & - \\
\hline
\end{tabular}

${ }^{\mathrm{a}}$ All areas in $\AA^{2}$; RT, room temperature. For adjusted comparisons, see Table 5. References: ${ }^{1}$ Lis et al. [15], ${ }^{2}$ Janiak et al. [13], ${ }^{3}$ Gruner et al. [61], ${ }^{4}$ Reiss-Husson [60], ${ }^{5}$ Small [62], ${ }^{6}$ Tardieu et al. [42], ${ }^{7}$ McIntosh et al. [64], ${ }^{8}$ Rand and Parsegian [1], ${ }^{9}$ Koenig et al. [63], ${ }^{10}$ Sun et al. [4], ${ }^{11}$ Nagle et al. [3], ${ }^{12}$ Petrache et al. [36], ${ }^{13}$ Tristram-Nagle et al. [38], ${ }^{14}$ Büldt et al. [10], ${ }^{15}$ Wiener and White [25],

${ }^{16}$ Lewis and Engelman [12]. 


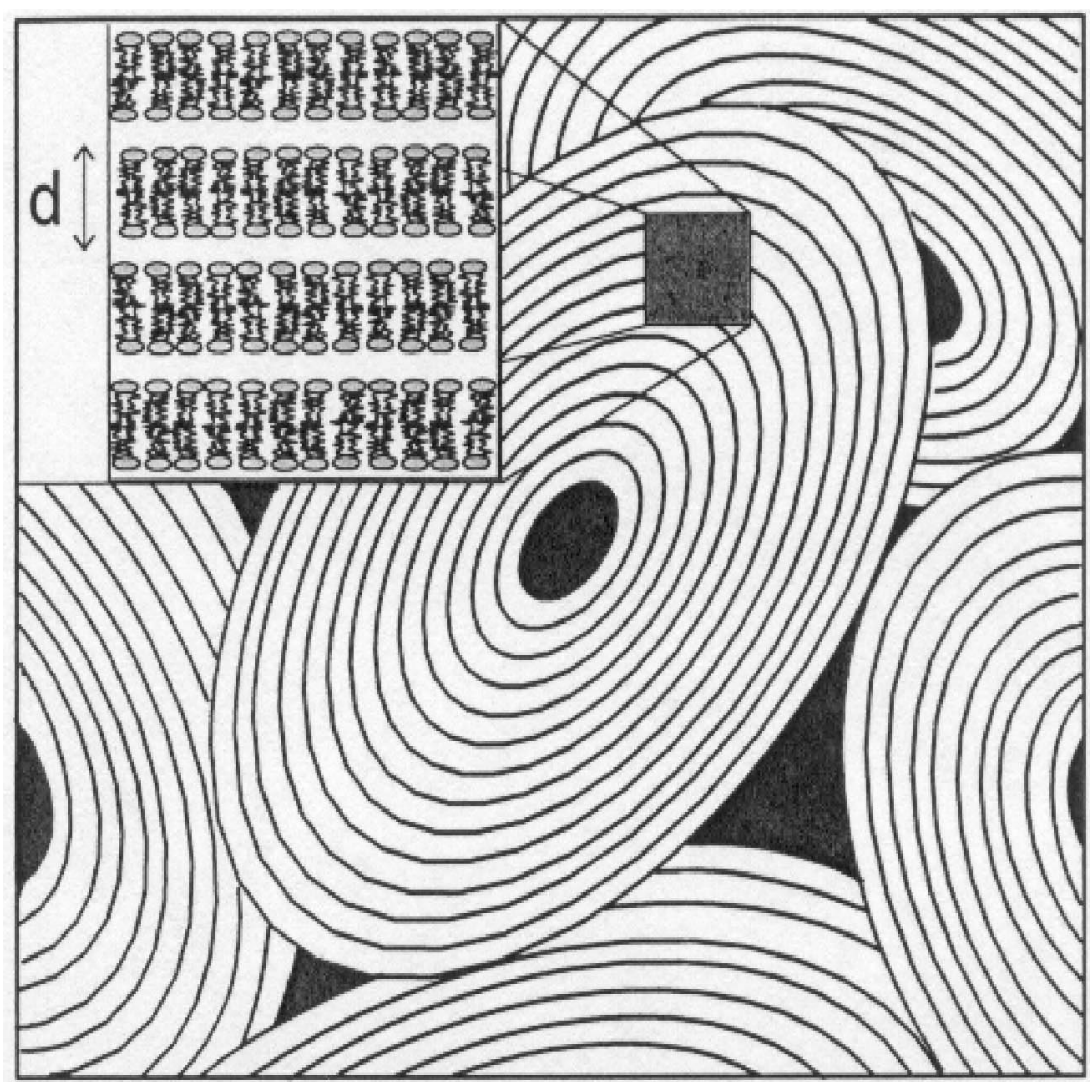

Fig. 3. Schematic view of MLVs with defect regions of excess water. Figure reproduced from [63] with permission of the authors.

simply by equating the geometric volume of the unit cell in a stack of bilayers, indicated in Fig. 2c, with the volumes of the lipid and water contained therein, namely,

$A D=2\left(V_{\mathrm{L}}+n_{\mathrm{W}} V_{\mathrm{W}}\right)$

Since $D, V_{\mathrm{L}}$ and $V_{\mathrm{W}}$ are all precisely measurable, one simply weighs the amount of water and the amount of lipid to obtain the number of waters/lipid $n_{\mathrm{W}}$, using the known molecular masses, and then $A$ follows directly. To obtain the fully hydrated value of $n_{\mathrm{W}}^{\mathrm{FH}}, D$ is measured as a function of $n_{\mathrm{W}}$ to find the value $n_{\mathrm{W}}^{\mathrm{FH}}$ for which further addition of water results in no further increase in $D$. Thermodynamically, this is the point where a two phase region is entered where the second phase is an excess water phase. (This is typical behavior for neutral lipids in MLVs. $D$ for charged lipids may increase indefinitely with increased water - such behavior is described as unbound bilayers in contrast to bound neutral bilayers.) Some results for $A$ using the GX method are listed in Table 3.
The reliability of the GX method has been repeatedly questioned $[26,41,63,65-68]$. One indication that there was a problem with the GX method was that different studies often came up with different values of $n_{\mathrm{W}}^{\mathrm{FH}}$. Even for the gel phase of DPPC the value of $n_{\mathrm{W}}$ ranged from 14 [46] to 19 [69] and the spread for fluid phase DPPC was from 23 [69] to 38 [15]. Some of this variation is correlated with the variation in $D$ noted in Section 3.2.1. Another is deciding the value of $n_{\mathrm{W}}^{\mathrm{FH}}$ beyond which more water does not increase $D$. It is also essential that the weighed lipid be dry and that none of the weighed water evaporates. In addition to these experimental issues, there is an intrinsic problem. Not all the water that is added to the lipid goes neatly between well defined stacks of bilayers with uniform spacings $D$. Indeed, the sketch in Fig. 3, showing MLVs as more or less spherical objects, indicates that there must be extra space between the MLVs. This extra volume must be occupied by water in addition to that which is included in the $n_{\mathrm{W}}$ value in Eq. 1. Other kinds of defect regions would also tend to include a larger proportion of 
water to lipid. All this water is measured by the gravimetric method, but not all of it should be included in the $n_{\mathrm{W}}$ value appropriate for Eq. 1 since it does not contribute to the measured $D$-spacing which reports the well-stacked portions of the sample. Defect regions, which do not affect $D$, have been visualized with electron microscopy and shown to become more prevalent near full hydration [67].

This discussion suggests that the GX method would obtain less water outside the stacks of bilayers if the samples were well annealed to reduce defect regions. Better annealed samples would give smaller and more reliable values of $n_{\mathrm{W}}^{\mathrm{FH}}$. Some of the differences in GX results could be due to different extents of annealing. From the preceding paragraph, one would also expect that the $n_{\mathrm{W}}$ that should appear in Eq. 1 is less than what is weighed, so the GX method would systematically overestimate $A$. Indeed, the GX results in Table 3 are consistently on the high side, although exceptions have occurred $[13,64]$.

\subsection{Corrected gravimetric X-ray method ( $G X C)$}

Another indication that the GX method is defective was that the results indicated that $A$ increases too strongly as the limit of full hydration is approached $[42,63]$. We now realize that much of this increase is due to the increase in the volume of defect regions which causes $n_{\mathrm{W}}$ to increase anomalously, especially near full hydration. However, there is also a real reason for an increase in $A$ with increasing hydration, as was emphasized by Rand and Parsegian [1]. Less than full hydration is equivalent to exerting osmotic pressure $P$ to remove water from the bilayers. The most obvious effect of osmotic pressure is to decrease the water space $D_{\mathrm{W}}$ and thereby the $D$ space. A second, more subtle effect is that osmotic pressure also decreases $A$ because this too extracts water from stacks of bilayers. This is most easily seen by examining the left side of Fig. $2 \mathrm{c}$ where the box labelled $\mathrm{H}_{2} \mathrm{O}$ corresponds to the volume of water, which can be reduced either by reducing $D-D_{\mathrm{B}}$ or by reducing $A$. The appropriate formula to describe this second effect follows from the definition of lateral compressibility, and can be written as [1]

$A=A_{0}-A D_{\mathrm{W}} P / K_{\mathrm{A}}$, where $A_{0}$ is the fully hydrated area when $P=0, K_{\mathrm{A}}$ is the phenomenological area modulus, and $D_{\mathrm{W}} P$ is the effective lateral pressure. However, while $A$ should increase to $A_{0}$ as full hydration $(P=0)$ is approached, Rand and Parsegian [1] realized that the changes in $A$ obtained from the unadulterated gravimetric method became much too large near full hydration for the values of $K_{\mathrm{A}}$ measured independently on giant unilamellar vesicles by the aspiration pipette method of Evans [70].

Realizing the difficulty with the GX method, Rand and Parsegian proposed to modify it by using measured values of the lateral area compressibility $K_{\mathrm{A}}$ [1]; we call this the GXC method, where the ' $\mathrm{C}$ ' signifies a compressibility correction. The idea, consistent with electron microscopy [67], is that the defect volumes become proportionately smaller as osmotic pressure is increased. It makes sense that it is easier to shrink the defect regions than it is to remove water from between the more closely packed bilayers. Rand and Parsegian used gravimetric values of $A$ obtained under osmotic pressure at 10 atmospheres and they then used Eq. 2 to extrapolate to fully hydrated $P=0$.

The results for $A$ from the GXC method [1] shown in Table 3 are significantly smaller than from the GX method. The fact that they are still on the high side could be due to residual amounts of defect water still remaining at $P=10 \mathrm{~atm}$. Also, when these results were published, $K_{\mathrm{A}}$ had only been measured for a few lipid bilayers and the extended tables of structural results in [1] used those few values for many other lipid bilayers. Recently, $K_{\mathrm{A}}$ has been reported for more lipids [17]. Furthermore, there has been a dramatic increase in the reported values of $K_{\mathrm{A}}$, for example, from $145 \mathrm{dyn} / \mathrm{cm}$ to $234 \mathrm{dyn} / \mathrm{cm}$ for DMPC [17]. This correction, which tends to decrease the previous values of $A$, is made in Section 7 .

\section{Electron density profile (EDP) method}

\subsection{Head-head thickness $D_{H H}$}

The gravimetric X-ray methods only use unit cell information from X-ray diffraction. For fluid phase bilayers this is just $D$, which comes from indexing the orders of low-angle diffraction. The EDP method in 
this section uses information about the structure within the unit cell. For fluid phase bilayers this is the electron density profile $\rho^{*}(z)$ (see Fig. $2 b$ ), given by

$\rho *(z)-\rho_{\mathrm{W}}^{*}=\frac{1}{D} F_{0}+\frac{2}{D} \sum_{h=1}^{h_{\max }} \alpha_{\mathrm{h}}\left|F_{\mathrm{h}}\right| \cos \left(\frac{2 \pi h z}{D}\right)$,

where $\rho_{\mathrm{W}} *$ is the electron density of water. For the different diffraction orders $h>0, \alpha_{\mathrm{h}}$ is the phase factor which can only assume values of +1 or -1 for centrosymmetric bilayers, and $F_{\mathrm{h}}$ is the bilayer form factor. $F_{\mathrm{h}}$ is often called the structure factor, but this name is also used for a completely different quantity to be discussed shortly. The discrete form factor samples the continuous single bilayer form factor

$F\left(q_{\mathrm{z}}\right)=\int_{-\infty}^{\infty}\left(\rho_{1} *(z)-\rho_{\mathrm{W}} *\right) \cos \left(z q_{\mathrm{z}}\right) d z$

at values of $q_{z}=2 \pi h / D, h=1,2, \ldots$, where $\rho_{1} *(z)$ is the electron density of a single bilayer. The continuous form factor accounts for the statistical distribution of electrons in the bilayer much like the atomic form factor accounts for the statistical distribution of electrons in an atom. The discrete bilayer form factor $F_{\mathrm{h}}$ is routinely obtained from the intensity $I_{\mathrm{h}}=F_{\mathrm{h}}^{2} / C_{\mathrm{h}}$ under the $h$ th diffraction peak. $C_{\mathrm{h}}$ is the Lorentz polarization correction factor; for low-angle scattering $C_{\mathrm{h}}$ is nearly proportional to $h^{2}$ for unoriented MLV samples and to $h$ for oriented samples. The zeroth order form factor $F(0)$ is given by [71]

$A F_{0}=2\left(n_{\mathrm{L}}^{*}-\rho_{\mathrm{W}}^{*} V_{\mathrm{L}}\right)=2\left(\rho_{\mathrm{L}}^{*}-\rho_{\mathrm{W}}^{*}\right) V_{\mathrm{L}}$

where $A$ is the area per lipid, $n_{\mathrm{L}} *$ is the number of electrons in the lipid molecule, $V_{\mathrm{L}}$ is the lipid molecular volume and $\rho_{\mathrm{L}} * \equiv n_{\mathrm{L}} * / V_{\mathrm{L}}$ is the average electron density of the lipid molecule. The form factors $F_{\mathrm{h}}$ involve an unknown scale factor, so only the absolute ratios $r_{\mathrm{h}}=\left|F_{\mathrm{h}} / F_{1}\right|$ of form factors are measured directly and this means that only relative electron density profiles are routinely reported. Obtaining absolute electron density profiles is discussed in Section 5.6.

The most useful quantitative information from the electron density profile is the bilayer thickness $D_{\mathrm{HH}}$ (see Fig. 2b). $D_{\text {HH }}$ can generally be obtained to within a few $\AA$ provided that at least four orders $\left(h_{\max }=4\right)$ of diffraction are available. Nevertheless, even with four orders, the measured $D_{\mathrm{HH}}$ is subject to a Fourier truncation error. This error depends systematically upon the ratio $D_{\mathrm{HH}} / D$, as was verified by using fourth order Fourier reconstructions of reasonable model electron density profiles to determine the apparent value of $D_{\mathrm{HH}}$ with varying values of $D$ $[38,72]$. The ratio $D_{\mathrm{HH}} / D$ increases with increasing osmotic pressure $P$ because water is removed which decreases $D$. (Increasing $P$ also increases $D_{\mathrm{HH}}$ because $A$ decreases according to Eq. 2.) To estimate the correction to $D_{\mathrm{HH}}$, an electron density model is used [39] that was shown to adequately represent the results of several simulations [3].

\subsection{Bootstrap from gel phase}

McIntosh and Simon [73] introduced a method to use $D_{\mathrm{HH}}$ to obtain $A$ for the $L_{\alpha}$ phase. The idea is to use the much better determined gel phase and to use measured differences to extrapolate from gel phase structure to the $L_{\alpha}$ phase structure. The $L_{\alpha}$ phase area $A^{\mathrm{F}}$ is obtained in terms of the decrease in bilayer thickness $\Delta D_{\mathrm{HH}}=D_{\mathrm{HH}}^{\mathrm{F}}-D_{\mathrm{HH}}^{\mathrm{G}}$, the measured lipid volume $V_{\mathrm{L}}^{\mathrm{F}}$ and gel phase values for the hydrocarbon thickness $\mathrm{D}_{\mathrm{C}}^{\mathrm{G}}$ and headgroup volume $V_{\mathrm{H}}^{\mathrm{G}}$,

$A^{\mathrm{F}}=\frac{V_{\mathrm{L}}^{\mathrm{F}}-V_{\mathrm{H}}^{\mathrm{G}}}{D_{\mathrm{C}}^{\mathrm{G}}+\Delta D_{\mathrm{HH}} / 2}$.

This method was first applied to DLPE with the result $A_{\mathrm{DLPE}}^{\mathrm{F}}=51.2 \AA^{2}$ at $T=35^{\circ} \mathrm{C}[26,73]$. DLPE was a favorable first choice because the chains are perpendicular to the bilayer in the gel phase, so gel phase quantities are easier to obtain than for PCs where the chains are tilted. However, complete gel phase structure of DPPC has subsequently been obtained in the sense of Fig. 2c $[4,41]$. Another reason DLPE was more favorable than the PCs is that there were four orders of diffraction for fully hydrated $L_{\alpha}$ phase DLPE, but not for DPPC, and we now turn to this major hurdle.

\subsection{Why so few orders of diffraction?}

The immediate shortcoming of the electron density profile approach is that fully hydrated samples of many lipids, such as unoriented DPPC dispersions in the $L_{\alpha}$ phase, have only two robust orders of 
diffraction. Electron density profiles using two orders of diffraction are not sufficiently accurate, even for $D_{\mathrm{HH}}$. The simplistic explanation for so few orders is that fluctuations and disorder reduce higher order intensities. However, to make sense of diffraction data, it is necessary to understand that there are two quite different aspects of this general explanation.

Most of the analyses of electron density and neutron scattering length profiles implicitly assume that a stack of bilayers is a one dimensional crystal with a regular and uniform $D$ spacing. Disorder and local molecular fluctuations within each bilayer give rise to the broad component distribution functions in Fig. 2a which, in turn, mean that the electron density profile shown in Fig. $2 b$ is broad. Therefore, higher order terms $F_{\mathrm{h}}$ in the Fourier expansion are small, so the higher order peak intensities are small. This point, which has been made forcefully by Wiener and White [19], is, however, only the first part of the explanation for the absence of higher order diffraction peaks.

The second reason for the absence of higher order peaks is that stacks of lipid bilayers are not one dimensional crystals, but smectic liquid crystals. Smectic liquid crystals have large scale (long wavelength) fluctuations (see Fig. 4) that destroy crystalline long-range order and replace it with quasi-longrange-order (QLRO) in which pair correlation functions diverge logarithmically instead of remaining

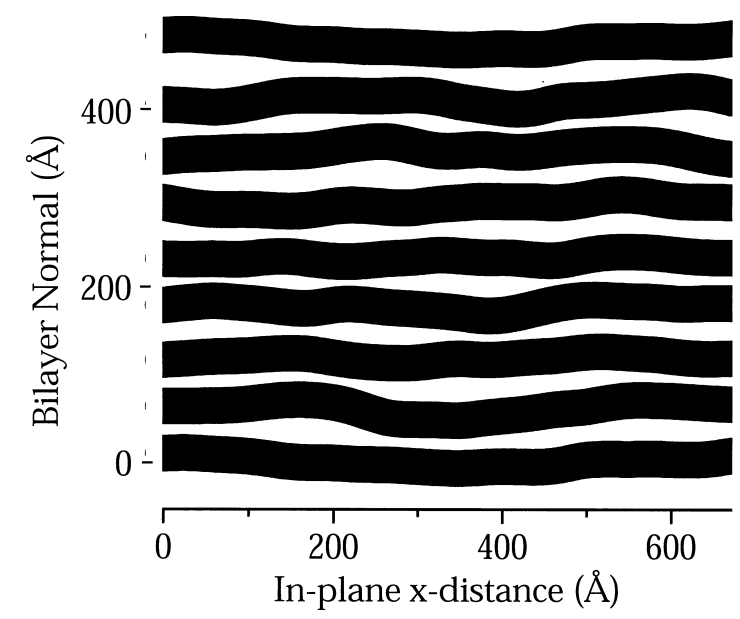

Fig. 4. Snapshot of fluctuations from a non-atomic level Monte Carlo simulation [91]. bounded as in crystals. Because long-range order is destroyed, Debye-Waller theory of scattering from crystals with lattice fluctuations is not appropriate (see appendix to [3]). Instead, QLRO changes the scattering peak shape from an intrinsic delta function by removing intensity from the central scattering peak and spreading it into tails of diffuse scattering centered on the original peaks. The magnitude of this shifting of intensity increases with increasing diffraction order. For high enough order $h$, the scattering peaks are completely converted to diffuse scattering even if the form factors $F_{\mathrm{h}}$ for the local lipid bilayer are large.

The preceding distinction between short-range and long-range fluctuations can be summarized as follows. Short-range fluctuations are intrinsic to the single lipid bilayer. These are the fluctuations that are studied in typical MD simulations. (The first exception has recently been reported [179].) They correspond to disorder within a unit cell in a crystalline stack of repeat units. In contrast, long-range fluctuations are fluctuations in the relative positions of the unit cells, which may be thought of as the centers of the bilayer. These longer range fluctuations, shown in a Monte Carlo simulation in Fig. 4, do not change the distribution functions of molecular components relative to the bilayer center, so they do not affect the structure of the single lipid bilayer.

Both kinds of fluctuations reduce the intensity of the higher orders. The first kind of fluctuations are local and their reduction in higher orders faithfully reflects the true bilayer structure. This is most easily seen by considering an electron density profile that consists of two symmetrically placed Gaussians

$\rho *(z)=\exp \left[-\left(\left(z-z_{0}\right) / \delta z\right)^{2}\right]+\exp \left[-\left(\left(z+z_{0}\right) / \delta z\right)^{2}\right]$

with widths $\delta z$, for which the form factor $F(q)$ is

$F(q)=\exp \left[-(q \delta z / 2)^{2}\right] \cos \left(q z_{0}\right)$.

The exponential factor in $F(q)$ decays more rapidly for higher orders (larger $q$ ) when the electron density has broader features (larger $\delta z$ ).

In contrast, the reduction in intensity due to the second kind of fluctuations comes about because of its effect on the stacking interference factor, which is 
often called the structure factor $S(q)$. The measured scattering intensity $I(q)$ is given by the product

$I(q)=S(q)|F(q)|^{2} / C_{\mathrm{h}}$.

In crystallography $S(q)$ is assumed to be essentially a delta function, so the $I(q)$ peaks are assumed to be narrow subject only to instrumental resolution broadening and perhaps finite sample size effects. Something quite different happens for smectic liquid crystals. The structure factor becomes intrinsically broader, so that intensity is removed from the peaks into the tails of $S(q)$ where it cannot be easily measured due to low intensity compared to background. This artifactually decreases the apparent intensity $I_{\mathrm{h}}$ and should be corrected since large scale fluctuations do not affect local bilayer structure.

This correction requires taking a rather different kind of data than conventional crystallography. The subsequent analysis uses liquid crystal theory, which is quite different from ordinary crystallographic analysis. A very appropriate name for this method is 'liquid crystallography'. This name, however, should not be confused with the same name that has been used by Wiener and White [25] in a series of papers that introduced a different major innovation that is reviewed in Section 8.2. Wiener and White properly emphasized that the first kind of molecular fluctuations within each unit cell are intrinsic to liquid crystals. However, this first kind of short-range disorder is also present in highly disordered solids and no particular properties of liquid crystals are used in the Wiener and White analysis. It is the second kind of long-range fluctuations that requires an analysis specifically tailored to liquid crystals that we suggest should be called 'liquid crystallography'.

\subsection{Liquid crystallography}

The beginning of liquid crystallography was a remarkably succinct three page paper by Caillé [74], communicated to the French Academy of Sciences by Guinier. That paper predicted power law tails on the diffraction peaks for smectic liquid crystals and it related the powers (exponents) to bulk phenomenological material properties, the bending modulus $K_{\mathrm{c}}$ and the bulk compression modulus $B$; the latter is a simple harmonic representation of the in- teractions between adjacent bilayers in a stack. The predictions of the theory were later verified by highly precise experiments on general smectics [75] and later on lipid bilayers [76].

Before Caillé's paper [74], Guinier [77] had elucidated the important distinction between disorder of the first and second kind, and emphasized that disorder of the second kind destroys crystalline longrange order. Applied to a one-dimensional stack of bilayers, Guinier's theory of disorder of the second kind is the same as the paracrystalline theory of Hosemann [78]. The Caillé theory [74] also treats fluctuations of the second kind, but it is considerably different from the earlier theories [77,78]. The earlier theories assumed that there is only stacking disorder. However, bilayers can also undulate so the local water spacing can vary with in-plane coordinates $(x, y)$ (Fig. 4). Another major distinction between the theories is that Caille's is based on a realistic Hamiltonian model rather than the purely stochastic approach of paracrystalline theory. However, the Caillé theory is considerably more difficult to apply, and paracrystalline theory has been effectively used for biomembranes [79], so it was appropriate to test whether Caillé theory really represents a significant improvement for lipid bilayers. Our group has documented the definite superiority of Caille theory for $L_{\alpha}$ phase DPPC bilayers [45]. On the other hand we have found that the scattering peaks are broader for the low temperature phases and appear not to follow the Caillé form, as was noted by Lemmich et al. [80]. This is consistent with the interpretation of McIntosh and Simon that the undulation fluctuations are much smaller for the low $T$ phases [81], so quite likely the disorder there is dominated by frozen-in defects that are not appropriately treated by the Caillé theory.

There are two main effects of liquid crystallography. The first is that the proportion of diffuse scattering to total scattering increases with order $h$. Indeed, for high enough $h$ the scattering is entirely diffuse and no central peak can be seen. The second is that the proportion of the scattering that is diffuse increases for all orders as the lipids become more fully hydrated, so the higher orders of diffraction disappear. Even the second-order $F_{2}$ for DPPC systematically falls off the continuous transform $F(q)$ obtained at $98 \%$ relative humidity $(\mathrm{RH})$ as the humidity is increased to full hydration [3]. These effects, 


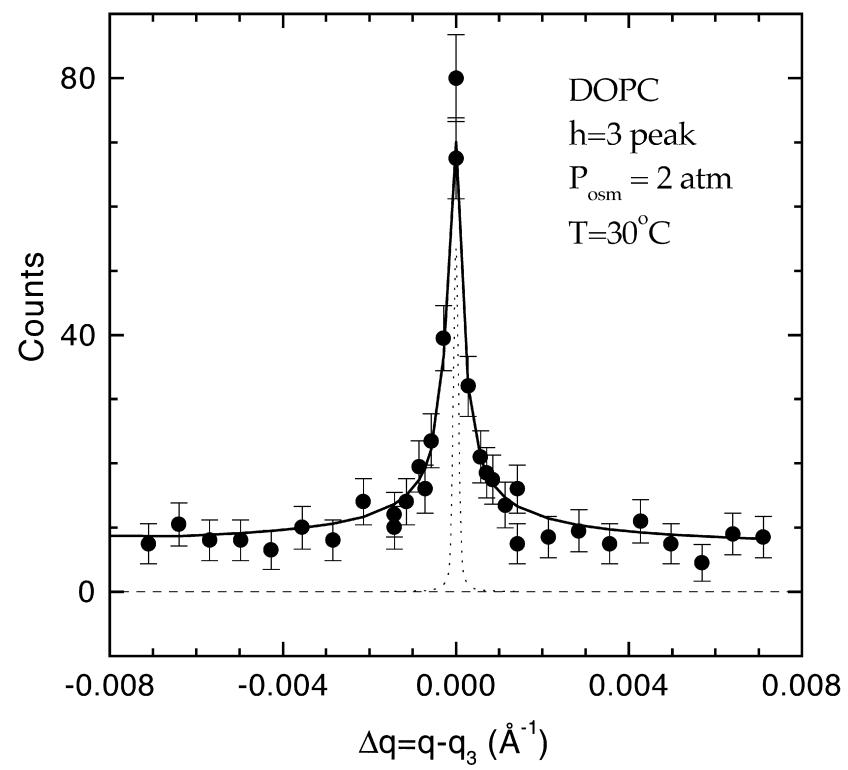

Fig. 5. Example of hidden diffuse scattering under $h=3$ peak in DOPC. The solid line is the fit that is determined by the first three orders of diffraction. The dashed horizontal line shows the background corrected baseline, drawn at zero counts. There is a large integrated intensity in the tails that extend halfway to the next peak which is located at $\Delta q=0.1 \AA^{-1}$. The dotted line shows the resolution function.

which were paradoxical in the context of conventional diffraction analysis, are fully predicted by liquid crystallography.

To carry out liquid crystallography the Caillé theory was improved beyond the prediction of power law tails to include quantitative amplitudes of the tails to the scattering peaks [82]. The ensuing modified Caille theory (MCT) enables prediction of the shapes of the scattering peaks for all orders using only a few parameters, primarily the average domain size $L$, which affects the width of the central peak, and the Caillé $\eta_{1}$ parameter [74],

$\eta_{1}=\frac{k_{\mathrm{B}} T}{8 \sqrt{B K_{\mathrm{c}}}} \frac{4 \pi}{D^{2}}$.

This $\eta_{1}$ parameter is also proportional to the mean square fluctuations $\sigma^{2}$ in the water space [47] and it governs the size of the scattering tails as well as the power law decay. To obtain the diffraction peak shapes experimentally, a silicon analyser crystal with instrumental resolution $\delta q=0.0001 \AA^{-1}$ was used [45]. However, with such high resolution, most scattered X-rays do not get to the detector, so a synchrotron source is helpful. By measuring suffi- ciently far into the power law tails before signal-tonoise becomes too small, the $\eta_{1}$ parameter can be obtained. It might be noted that the classic way of obtaining power law exponents such as $\eta_{1}$ is to use $\log -\log$ plots [75,76]. This is difficult because the range in $\Delta q=q-q_{\mathrm{h}}$ over which it is possible to measure straight line behavior on a log-log plot is limited to less than two decades. The small $\Delta q$ range is dominated by the sample domain/correlation size $L$ and the large $\Delta q$ range is limited by signal-to-noise and is further degraded by continuous changes in the form factor $F(q)$. In contrast, our method of analysis relies not only on the power law behavior, but also on the larger amplitudes in the tails when $\eta_{1}$ is larger. Once the parameters in the model have been obtained, the diffuse scattering that is in the tails of the structure factor $S(q)$ can be extrapolated. Even though this extrapolated diffuse scattering intensity is so small that it cannot be easily separated from background, the total amount of it is large because it extends all the way between scattering peaks. Fig. 5 indicates the amount of integrated intensity that is recovered using this extrapolation. When this hidden intensity is added, the result is that liquid crystallography does indeed predict the effects in the preceding paragraph quantitatively, and the use of it enables more accurate form factors $F_{\mathrm{h}}$ to be obtained that are true to the bilayer structure.

\subsection{Structural results}

The method we have been using to obtain structural results first obtains fluctuation corrected form factors for unoriented samples using liquid crystallography. Electron density profiles are drawn for those samples that have four orders of diffraction. Such samples are typically under osmotic stress of 20-50 atmospheres, corresponding to relative humidities of $98-96 \%$. To exert osmotic pressure we use the now classic method of Rand and Parsegian [1] to extract water from between bilayers using the polymers polyvinylpyrrolidone or dextran. There is never a problem choosing the first four or five phases for $F_{\mathrm{h}}$. For more orders, the continuous transform can be approximated by plotting $F\left(q_{\mathrm{h}}\right)$ obtained at many osmotic pressures as shown in Fig. 6. Then, fitting hybrid electron density models [39] to the intensities gives unambiguous higher order phases. For PC lip- 


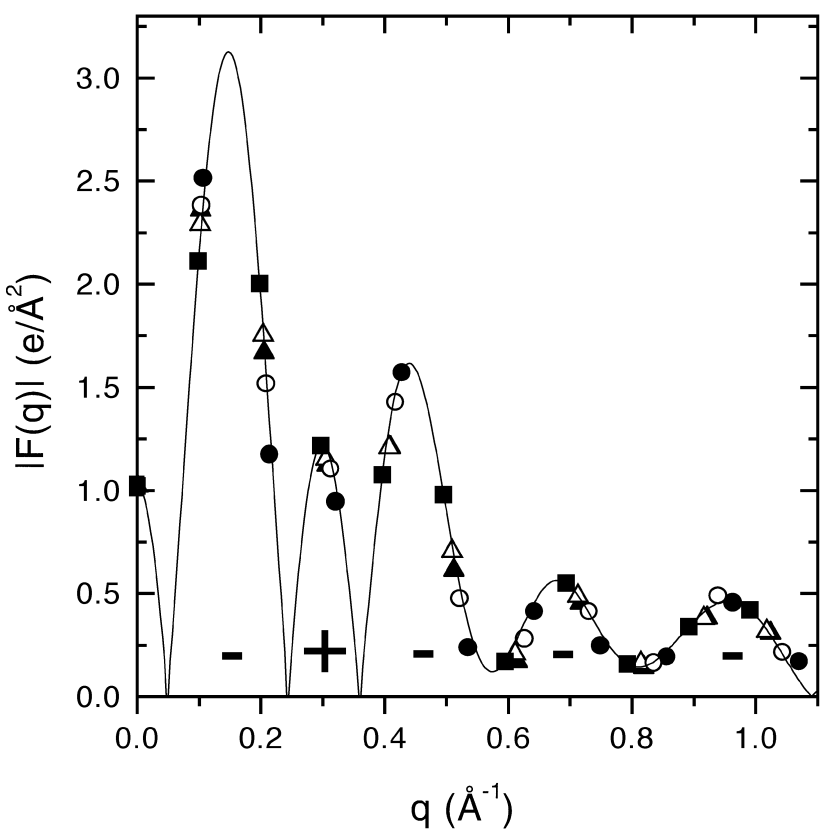

Fig. 6. The solid line shows the continuous transform $F(q)$ for fully hydrated gel phase DPPC. The data points show the discrete form factors $F_{h}$ for $h=1-10$ for five different values of $D$ from $58.7 \AA$ to fully hydrated $63.2 \AA$. The phase factors are indicated by the signs under each lobe. The first five phase factors are obvious. The next five require more detailed analysis $[39,49]$.

ids, we use Eq. 6 to obtain $A$ for various osmotic pressures $P$. The reference phase that we have used in Eq. 6 is the gel phase of DPPC, for which headgroup volume $V_{\mathrm{H}}^{\mathrm{G}}$ and hydrocarbon thickness $D_{\mathrm{C}}^{\mathrm{G}}$ are accurately known from gel phase studies $[4,41]$. Inspired by Eq. 2, we plot the ensuing values of $A$ against $A D_{\mathrm{W}} P$, where the slope is $-1 / K_{\mathrm{A}}$ and the intercept is the full hydration value $A_{0}$. To do this we also need the water thickness $D_{\mathrm{W}}$, which is obtained from the partitioning indicated in Fig. 2c, namely, $A D_{\mathrm{W}}=n_{\mathrm{W}} V_{\mathrm{W}}$, where $n_{\mathrm{W}}$ is obtained directly from Eq. 1 and the value of $A$. This is illustrated in Fig. 7 for data from DOPC [38]. Although the slope is not well determined by the data in Fig. 7, the best fit gives $K_{\mathrm{A}}=188 \mathrm{dyn} / \mathrm{cm}$ (solid line) [38] which may be compared to the more recently determined $K_{\mathrm{A}}=265 \pm 18 \mathrm{dyn} / \mathrm{cm}$ using the aspiration pipette method [17]. Literature results for $A$ using the EDP method are given in Table 3 . Corrections, such as those implied in Fig. 7 that take into account better $K_{\mathrm{A}}$ measurements, are made in Section 7.

\subsection{Absolute electron density profiles}

Obtaining absolute electron density profiles requires information in addition to low-angle scattering. Wide-angle X-ray studies of the gel phase and volumetric studies as a function of temperature give the molecular volumes of the lipid molecule $V_{\mathrm{L}}$ and some of its component groups, especially the methylenes $V_{\mathrm{CH}_{2}}$ and the terminal methyls $V_{\mathrm{CH}_{3}}$ in the chains $[26,29]$. From these volumes one obtains electron density information. This kind of information is better used with the hybrid electron density model [39] than with the Fourier representation (Eq. 3). The hybrid model combines constant density regions for the methylenes with Gaussians for the headgroups and the terminal methyl trough. The hybrid model has the additional advantage over the Fourier representation in that data for many samples, including different osmotic pressures and $D$ spacings can be used simultaneously to obtain the best fit if there is little change in structure.

If one fits any model to measured relative form factors, the model must contain an unknown scale factor $K$. One way to constrain $K$ in the hybrid model is to require that the model have the correct value for the electron density in the methylene plateau. Another way is to require that the methyl trough be the correct size to account for the known deficit

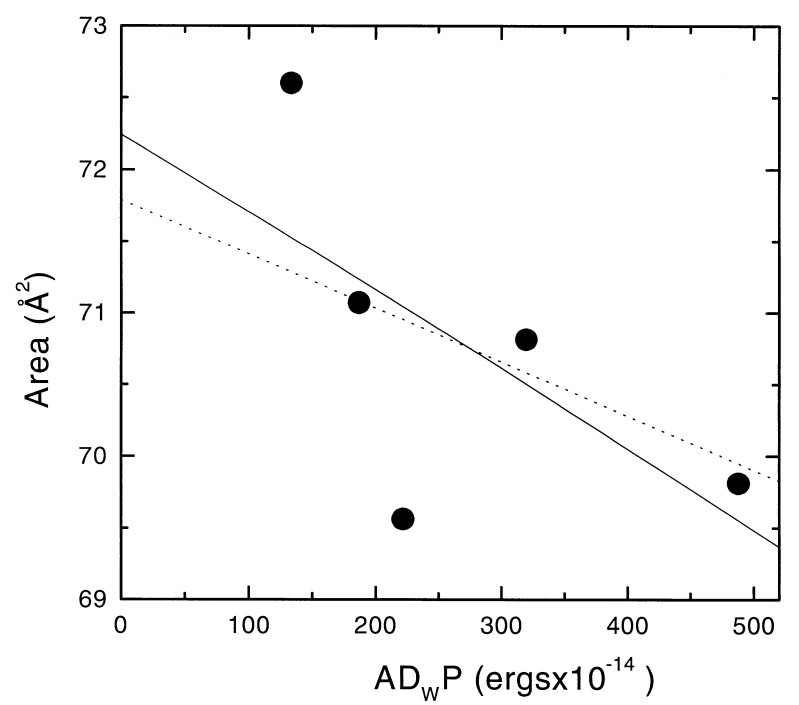

Fig. 7. Dependence of $A$ versus $A D_{\mathrm{W}} P$ for DOPC at $30^{\circ} \mathrm{C}$ [38]. The solid line is the best fit with slope $-1 / K_{\mathrm{A}}$ corresponding to $K_{\mathrm{A}}=188 \mathrm{dyn} / \mathrm{cm}$. The dotted line is the best fit using $K_{\mathrm{A}}=265$ $\mathrm{dyn} / \mathrm{cm}$ from [17]. 
of electron density in the terminal chain methyls. Yet another way to constrain $K$ is to require that the model has the value of $F(0)$ that is obtained from $V_{\mathrm{L}}$ and $A$ using Eq. 5. Although any one of these constraints should suffice in principle, in practice when only one or two are applied, the others are then not satisfied. It is therefore best to use all three constraints simultaneously [3]. This is not surprising or disturbing because the low-angle diffraction information is confined to low $q$, corresponding to $h=4$, so low-angle X-ray information should be supplemented as much as possible by other information.

The preceding, somewhat strenuous, method of constructing electron density profiles has only been applied to the DPPC $L_{\alpha}$ phase [3]. It has also been applied to the $L_{\beta}{ }^{\prime}$ phase, but with data only at full hydration [39]. Derivation of absolute electron density profiles for other PC lipids is based on this DPPC result supplemented by a simple argument. Since the headgroups are the same, the integrated electron density under the headgroup peak in excess of the level due to water on one side, and hydrocarbon on the other, should scale inversely with the area $A$, and the prefactor can be determined from $V_{\mathrm{H}}$ and the number of electrons in the headgroup $[36,38]$.

It might also be noted that one could contemplate using the scale for the electron density profiles provided by simulations. However, different simulations give rather different scaling factors (see Fig. 7 in [3]), so a more immediate use of absolute electron density profiles is to test simulations. Fig. $2 b$ indicates that the simulation result reported here passes this test.

\section{Interactions between bilayers}

The preceding section shows that long-range fluctuations of the second kind complicate the task of obtaining average structure of lipid bilayers in the highly fluctuating, fully hydrated $L_{\alpha}$ phase. From a structural point of view these fluctuations have no intrinsic value. We now turn to a topic where these fluctuations do have intrinsic importance that is directly addressed by liquid crystallography. Since our review of this topic will be somewhat brief, the reader may wish to consult a fuller review of the recent literature [83].

\subsection{Fundamental interactions}

It was originally shown by Helfrich [84] that undulation fluctuations cause an effective interaction between lipid bilayers, the fluctuation interaction. The conceptual basis for this interaction is that two bilayers close to one another cannot fluctuate as much as two bilayers far from each other. Mutual suppression of independent fluctuations leads to a decrease in entropy which increases the free energy $F$ as the average water separation distance $D_{\mathrm{W}}{ }^{\prime}$ is decreased, so this interaction is repulsive and entropic. It is an entropic energy (-TS) that is absent at absolute zero temperature, rather than a bare energetic interaction (E).

Helfrich showed that, when the only bare energetic interaction between bilayers is steric (excluded volume interaction), the form of the effective fluctuation free energy is [84]

$F_{\mathrm{fl}}=0.42 \frac{\left(k_{\mathrm{B}} T\right)^{2}}{K_{\mathrm{c}} D_{\mathrm{W}}^{\prime 2}}$.

This result has been confirmed experimentally in those systems in which the bare interaction between non-fluctuating bilayers can be closely approximated as zero over most of the relevant range in water spacing $D_{\mathrm{W}^{\prime}}$ [85]. Such systems are described as being in the hard confinement regime because the bare potential can be thought of as confinement of each bilayer between hard walls formed by neighboring bilayers. However, for lipid bilayers in typical MLVs there are additional bare interactions besides the steric interaction. If these interactions have ranges that are comparable to the average water spacing $D_{\mathrm{W}}{ }^{\prime}$, then the approximation of the bare interaction $V_{\mathrm{B}}\left(D_{\mathrm{W}}{ }^{\prime}\right)$ by a hard box-like potential is obviously deficient. It is then appropriate to consider a soft confinement regime [70,86,87].

One important bare interaction is the strong repulsive hydration force which, even though not so well understood, has been well documented experimentally $[1,88,89]$ to have the form

$$
V_{\text {hyd }}\left(D_{\mathrm{W}}{ }^{\prime}\right)=P_{\mathrm{h}} \lambda_{\mathrm{h}} \mathrm{e}^{-D_{\mathrm{w}^{\prime}} / \lambda_{\mathrm{h}}},
$$

with parameters $\lambda_{\mathrm{h}}$ (decay length) and prefactor $P_{\mathrm{h}}$. Another important bare interaction is the van der 
Waals attractive interaction,

$$
\begin{aligned}
& V_{\mathrm{vdW}}\left(D_{\mathrm{W}}{ }^{\prime}\right)= \\
& -\frac{H}{12 \pi}\left(\frac{1}{D_{\mathrm{W}}^{\prime 2}}-\frac{2}{\left(D_{\mathrm{W}^{\prime}}+D_{\mathrm{B}}{ }^{\prime}\right)^{2}}+\frac{1}{\left(D_{\mathrm{W}^{\prime}}+2 D_{\mathrm{B}}{ }^{\prime}\right)^{2}}\right)
\end{aligned}
$$

where $H$ is the Hamaker parameter. This is the interaction assumed to be responsible for limiting the swelling in bilayers composed of lipids with no net charge. We define $D_{\mathrm{W} 0}{ }^{\prime}$ to be the limiting water space for fully hydrated MLVs with osmotic pressure $P=0$. Because $D_{\mathrm{W} 0}{ }^{\prime}$ is only $10-30 \AA$, a graph of bare potential $V_{\mathrm{B}}$ versus $D_{\mathrm{W}}{ }^{\prime}$ on this length scale shows considerable variation. For charged lipids in low salt, one should also consider an electrostatic interaction, but this is absent for the neutral lipids. An additional very short-range repulsion has been measured and attributed to headgroup protrusions [90]. We do not include it since it only plays a role for lipids under high osmotic pressure and small water space $D_{\mathrm{W}}{ }^{\prime}$. It does, however, play the formal role of suppressing the singularity in the van der Waals potential at $D_{\mathrm{W}^{\prime}}=0$.

It has been proposed for the soft confinement regime that the fluctuation interaction free energy in Eq. 11 should be modified [86,87] and a formula

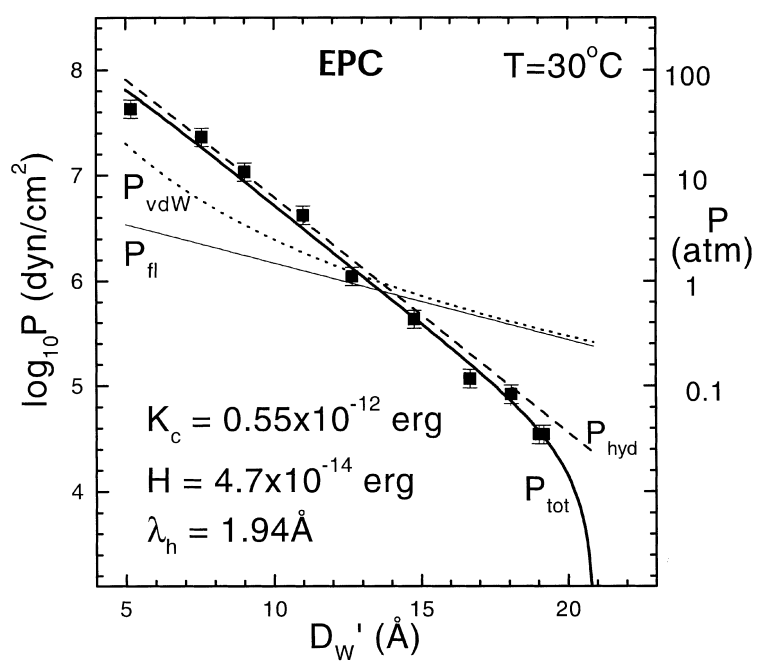

Fig. 8. Osmotic pressure $P$ versus steric water space $D_{\mathrm{W}}{ }^{\prime}$. Various lines show contributions from various interactions, with the bold solid curve showing the fitted total $P$.

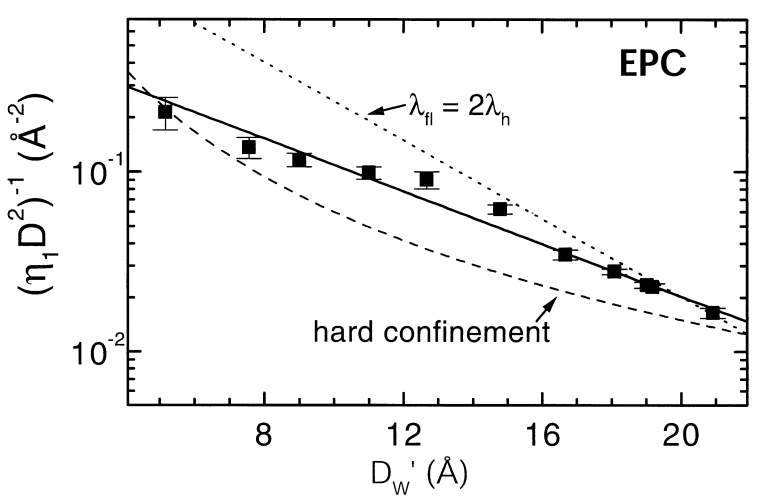

Fig. 9. Functional form of fluctuation free energy versus water spacing is represented by an exponential with decay length $\lambda_{\mathrm{fl}}=5.9 \AA=3 \lambda_{\mathrm{h}}$ (solid line).

involving an exponential with decay length $\lambda_{\mathrm{fl}}$

$F_{\mathrm{fl}}=\left(\pi k_{\mathrm{B}} T / 16\right)\left(P_{\mathrm{h}} / K_{\mathrm{c}} \lambda_{\mathrm{h}}\right)^{1 / 2} \exp \left(-D_{\mathrm{W}}{ }^{\prime} / \lambda_{\mathrm{fl}}\right)$

has been offered [70,87]. This exponential functional form is quite different from the power law form in Eq. 11 established for the hard confinement regime. Furthermore, the decay length $\lambda_{\mathrm{fl}}$ was predicted to be twice the decay length $2 \lambda_{\mathrm{h}}$ of the hydration force [70,87].

For lipid bilayers the now traditional way [1] to investigate interbilayer forces experimentally is to measure the average water space $D_{\mathrm{W}}{ }^{\prime}$ as osmotic pressure $P$ is varied; such data are usually plotted as $\log P$ as in Fig. 8. The data clearly show an exponential increase for $P$ greater than 10 atmospheres and this is the experimental basis for the force that is named the hydration force. However, to fit the data over all $P$ there are at least three energies involved with four parameters $\left(\lambda_{\mathrm{h}}, P_{\mathrm{h}}, H\right.$ and $\left.K_{\mathrm{c}}\right)$. There are also different ways to define water space (gravimetric $D_{\mathrm{W}}$ [1] versus steric $D_{\mathrm{W}^{\prime}}$ [51] - see Fig. 2c). While it has been encouraging that fits to the $P$ data make sense with reasonable values for the parameters [88], there are too few data to provide fits that uniquely separate $P$ into its constituent forces. As noted by Parsegian and Rand [28], “... dissection of the measured pressure $P$ into its physically distinct components is a problem almost as difficult as the theoretical explanation of these components themselves". In particular, the functional form of the fluctuation pressure is an important assumption in carrying out such fits. 


\subsection{Experimental window on the fluctuation force}

Experimental study of the fluctuation correction for structural studies provides an experimental window on the fluctuational force. The most direct connection is that the fluctuational free energy $F_{\mathrm{fl}}$ is related to the Caille $\eta_{1}$ parameter [47] by

$F_{\mathrm{fl}}=\left(\frac{k_{\mathrm{B}} T}{2 D}\right)^{2} \frac{1}{K_{\mathrm{c}} \eta_{1}}$.

The bending modulus $K_{\mathrm{c}}$ is defined to be a property only of the single, isolated bilayer, so the functional form of $F_{\mathrm{fl}}$ can be obtained from $\eta_{1}$ and $D$. A plot of $1 / \eta_{1} D^{2}$ therefore shows the functional form of $F_{\mathrm{fl}}$. Data for EPC are shown in Fig. 9. Data for DPPC, DMPC, EPC [47] and DOPC [38] are all inconsistent with the hard confinement functional form in Eq. 11, proving that a theory of soft confinement is necessary. The data are consistent with the prediction of the soft confinement theory that the fluctuation free energy has an exponential decay with $D_{\mathrm{W}}{ }^{\prime}$. However, the effective decay length of the fluctuation free energy, which is defined to be $\lambda_{\mathrm{fl}}$, is consistently larger than the theoretical prediction $\lambda_{\mathrm{fl}}=2 \lambda_{\mathrm{h}}$, shown by the dotted line in Fig. 9 . Also for DOPC, DPPC and DMPC the experimental ratio $\lambda_{\mathrm{fl}} / \lambda_{\mathrm{h}}$ is in the range $2.5-3$.

Simulations have been performed to address the issue from the preceding paragraph concerning the experimental result that $\lambda_{\mathrm{f} 1} / \lambda_{\mathrm{h}}$ is consistently greater than the theoretical prediction of 2 . This result could have been due to several reasons, including: (i) the analytical soft confinement theory (Eq. 14) may be inaccurate, (ii) the bare interactions may be inadequately described by the harmonic approximation in the Caille theory or (iii) there may be experimental artifacts. By doing a simulation with the same form of the interactions as in Eqs. 12 and 13, (ii) and (iii) were bypassed and (i) was tested directly. The result of the simulation is that $\lambda_{\mathrm{f}} / \lambda_{\mathrm{h}}$ is about 2.4 [91]. Although this is a bit smaller than the experimental ratio, it clearly agrees with the experimental conclusion that the ratio is larger than the value of 2 given by Eq. 14. This lends confidence to the experimental results. It should also be emphasized that, while it is always desirable to develop analytical theory and the result has been insightful [87], the problem is very difficult, so that analytic theory necessarily involves uncontrollable (mean field type) approximations that can and should be tested, especially when numerical accuracy is required.

\subsection{Determination of interbilayer interaction parameters}

The thermodynamic quantities of greatest interest are the osmotic pressure $P$ and the root mean square fluctuation $\sigma$ in water spacing, both as a function of mean interbilayer spacing $D_{\mathrm{W}^{\prime}}$. $\sigma$ is simply related to the measured Caillé $\eta_{1}$ parameter [47] by

$\sigma^{2}=\eta_{1} D^{2} / \pi^{2}$.

Simulation results $[91,92]$ compare favorably with the analytic theory [87] for small $D_{\mathrm{W}^{\prime}}$ and when there are no van der Waals interactions, but the discrepancy grows as $D_{\mathrm{W}^{\prime}}$ approaches full hydration where $P=0$. These discrepancies are too large to ignore when trying to fit data to determine interaction parameters.

The basic experimental approach [47] determined the decay length $\lambda_{\mathrm{fl}}$ of the fluctuation force and its magnitude up to a factor of the bending modulus $K_{\mathrm{c}}$. Assuming a value of $K_{\mathrm{c}}$, fits to the bare pressure $P_{\text {bare }}=P-P_{\mathrm{fl}}$ gave well determined values for $H_{\text {Hamaker }}, \lambda_{\mathrm{h}}$ and $P_{\mathrm{h}}$. However, fits with different values of $K_{\mathrm{c}}$ over the range spanned by literature values gave equally good fits, essentially because variations in $H$ compensated for variations in $K_{\mathrm{c}}$ whereas values of $\lambda_{\mathrm{h}}$ (about $2 \AA$ ) and $P_{\mathrm{h}}$ were robustly determined [47]. This approach used the fluctuation data $\eta_{1}$ only to eliminate the effective modulus $B$ for interbilayer interactions and this throws away information when doing the final fit to the bare interaction parameters. Simulations, however, give both $P$ and $\eta_{1}$. Requiring both to agree with both sets of data is a stronger constraint on the interaction parameters. Detailed fits of simulations and data have not yet been carried out. However, for DMPC at $30^{\circ} \mathrm{C}$ the following parameter set fits both $P$ and $\eta_{1}$ fairly well over the full range of $D_{\mathrm{W}^{\prime}}$ [93]: $H=7.13 \times 10^{-14} \mathrm{erg}$, $K_{\mathrm{c}}=0.5 \times 10^{-12} \mathrm{erg}, \lambda_{\mathrm{h}}=1.91 \AA$ and $P_{\mathrm{h}}=1.32 \times 10^{9}$ $\mathrm{erg} / \mathrm{cm}^{2}$ and it is clear that larger values of $K_{\mathrm{c}}$ provide inferior fits. This value of $K_{\mathrm{c}}$ agrees well with [94], but it is smaller than the value given by [95]. The value of $H$ is somewhat larger than preferred by [96]. However, more lipid systems should be carefully 
analyzed before drawing definitive conclusions for these parameter values.

The present determination of parameter values does allow an important conclusion to be drawn, namely, that interactions between fully hydrated MLVs have negligible effect on the intrinsic structure of the lipid bilayer. Although this might seem to be obvious since the net force between fully hydrated bilayers is automatically zero, the fluctuation force is entropic (statistical) in nature, so the net bare forces are non-zero. However, for PCs with $D_{\mathrm{W}}{ }^{\prime}$ greater than $10 \AA$, the net interbilayer interaction energy per lipid molecule is less than $k T / 20$. This is negligible compared to the enthalpy of the main structural phase transition which is of order $15 \mathrm{kT}$. Another comparison is provided by Fig. 8 which shows that the bare interaction pressure is of order $0.25 \mathrm{~atm}$ at full hydration; using Eq. 2 with this pressure suggests that fully hydrated MLVs should have an area that is less than $0.02 \AA^{2}$ different from non-interacting unilamellar bilayers.

\section{Corrections and adjustments to A}

In this section we return to bilayer structure and perform three modifications to the literature values for $A$ in Table 3. The first and simplest adjustment is motivated by the desire to compare the $A$ values obtained by the different methods at a common temperature. This adjustment is easily made using the area thermal expansivity $\alpha=(1 / A)(\partial A / \partial T)_{\pi}$ which has been measured for giant unilamellar vesicles of several lipids [70]. Based on those results we use a value of $0.003 /{ }^{\circ} \mathrm{C}$ for most lipids. However, larger values are indicated for lipids near their main transitions and we use values of $\alpha$ in the range 0.003 $0.006 /{ }^{\circ} \mathrm{C}$ for DMPC in the range $24-30^{\circ} \mathrm{C}$ and for DPPC in the range $42-50^{\circ} \mathrm{C}$.

The second modification is to use recently reported values of the area compressibility modulus $K_{\mathrm{A}}$ which are obtained using the aspiration pipette method [17]. The new 'true' values of $K_{\mathrm{A}}$ are considerably larger than the older, 'apparent' values. This distinction, which involves the difference between using projected areas onto an average bilayer plane for the apparent $K_{\mathrm{A}}$ versus using actual local areas for the true $K_{\mathrm{A}}$, was made some time ago [94]. However, true $K_{\mathrm{A}}$
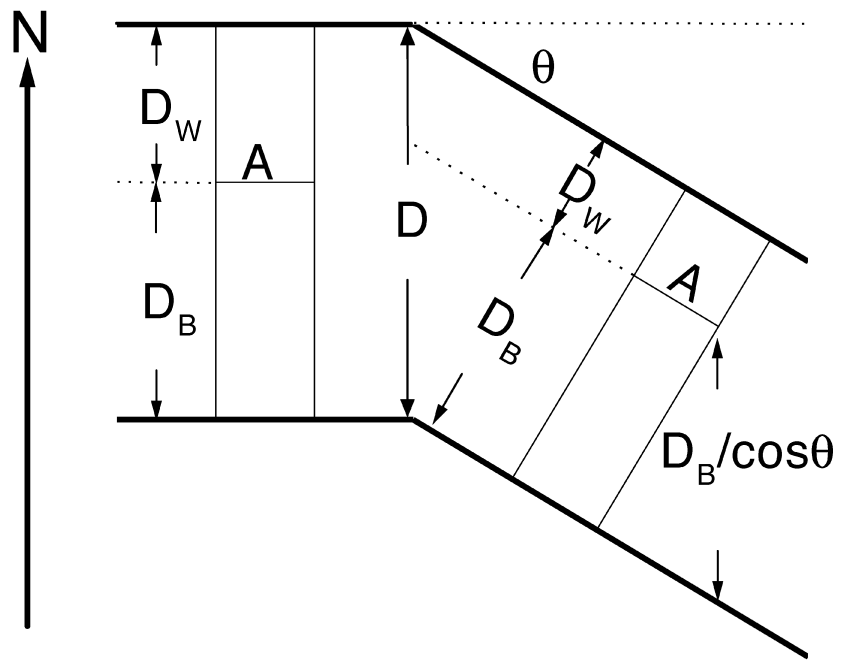

Fig. 10. Schematic of two sections of a fluctuating bilayer (plus associated water) in an MLV. The left section has its local normal along the average normal $N$ and the right section is tilted by angle $\theta$. A local unit cell is drawn in each section. The local thickness $D_{\mathrm{B}}+D_{\mathrm{W}}$ is given by $D \cos \theta$.

values were not given and most workers, including ourselves, have not appreciated this subtlety, and values of the true $K_{\mathrm{A}}$ have now been given for the first time [17]. This second correction, acting alone, reduces $A$ using the EDP method, as indicated in Fig. 7, and it reduces the previous GXC result for $A$. Before making this correction we first turn to the third correction that acts to increase $A$.

\subsection{New correction}

This correction involves undulation fluctuations in a different and additional way compared to how they were used in Section 5. The effect comes about from a simple geometrical consideration, illustrated in Fig. 10. On the two sides of the figure are two sections of a unit cell containing one bilayer. The section on the left is in the conventional orientation with the bilayer plane perpendicular to the bilayer stacking direction $N$. In order to illustrate the effect of undulations, the section on the right hand side of the figure is drawn tilted by $\theta$ with respect to the mean bilayer normal $N$. Of course, there are generally many different sections with a continuum distribution of tilt angles $\theta$ instead of just two sections with a discontinuous change in slope. Since the bilayer is contained in a stack of bilayers with mean repeat spacing $D$, there is the important constraint that the average vertical 
Table 4

Data used to make corrections

\begin{tabular}{|c|c|c|c|c|}
\hline Parameter & $\mathrm{DPPC}\left(50^{\circ} \mathrm{C}\right)$ & $\mathrm{DMPC}\left(30^{\circ} \mathrm{C}\right)$ & DOPC $\left(30^{\circ} \mathrm{C}\right)$ & $\operatorname{EPC}\left(30^{\circ} \mathrm{C}\right)$ \\
\hline$K_{\mathrm{C}}\left(10^{-12} \mathrm{erg}\right)$ & $0.5[47]$ & $0.56[17]$ & $0.80^{\mathrm{a}}[17,38]$ & $0.55[47,107]$ \\
\hline$\alpha\left({ }^{\circ} \mathrm{C}^{-1}\right)^{\mathrm{d}}$ & $0.003-0.006$ & $0.003-0.006$ & 0.003 & 0.003 \\
\hline$K_{\mathrm{A}}(\mathrm{dyn} / \mathrm{cm})$ & $250^{\mathrm{b}}$ & $234[17]$ & 265 [17] & $250^{\mathrm{b}}$ \\
\hline \multicolumn{5}{|l|}{$\xi(\AA)[38,47]$} \\
\hline (a) $P^{\mathrm{c}}=0$ & 76 & 53 & 70 & 72 \\
\hline$@ P=10$ & 28 & 21 & 33 & 28 \\
\hline$@ P=P_{\mathrm{MAX}}$ & 24 & 15 & 25 & 25 \\
\hline$P_{\mathrm{MAX}}$ & 23 & 27 & 56 & 29 \\
\hline \multicolumn{5}{|l|}{$\left\langle\theta^{2} / 2\right\rangle\left(\operatorname{radians}^{2}\right)$} \\
\hline (a) $P=0$ & 0.024 & 0.018 & 0.014 & 0.020 \\
\hline$@ P=10$ & 0.017 & 0.012 & 0.011 & 0.019 \\
\hline (a) $P=P_{\mathrm{MAX}}$ & 0.016 & 0.010 & 0.010 & 0.014 \\
\hline
\end{tabular}

${ }^{\text {a }}$ Temperature adjusted to $30^{\circ} \mathrm{C}$.

${ }^{b}$ Estimated, but see new results in text from Evans (private communication).

${ }^{c}$ All osmotic pressures are in atmospheres $\left(10^{6} \mathrm{dyn} / \mathrm{cm}^{2}\right)$.

${ }^{\mathrm{d}}$ Estimated from [70].

extent of the unit cell is the same $D$ for all sections. Of course, there are local fluctuations in $D$, but these are assumed to be uncorrelated with the undulations because of the overall stacking constraint. Now let us suppose for the moment that the mean thicknesses of the local bilayer, such as $D_{\mathrm{B}}$ and $D_{\mathrm{HH}}$ are the same in all sections, where both of these are measured perpendicular to the local bilayer. The form factor $F\left(q_{\mathrm{z}}\right)$ senses electron density along the average bilayer normal $N$, but along that direction, the actual head-head separation is $D_{\mathrm{HH}} / \cos \theta$. Therefore, the average apparent $D_{\mathrm{HH}}$ obtained from electron density profiles is larger than the local $D_{\mathrm{HH}}$. Correcting for this decreases the apparent $D_{\mathrm{HH}}$ and thereby increases the $A$ obtained by the EDP method in Eq. 6 . The GX and GXC areas are also affected, in a more subtle way as is discussed in subsection 7.2.

Let us now return to an unwarranted assumption made in the previous paragraph, namely, that $D_{\mathrm{B}}$ is the same in all sections. This assumption would then require that $D_{\mathrm{W}}$ be smaller in sections with larger $\theta$, but this would involve a reaction from the repulsive forces between adjacent bilayers that would tend to increase the local A. Stated differently, there is a competition between bilayer deformability and water space deformability. Near zero osmotic pressure the water space is much more deformable than the bi- layer and the assumption that $D_{\mathrm{B}}$ does not change is appropriate. However, we also need to consider $P \neq 0$ where many of the primary measurements of structure were performed. Fortunately, it turns out that this assumption essentially does not matter and that one obtains the same correction even when the bilayer deforms. The reason for this is only revealed by a derivation that minimizes the total free energy of the undulating system. We defer this derivation to subsection 7.3.

The primary quantity that is required to carry out this correction is $\langle 1 / \cos \theta\rangle$ where the angular brackets denote averages over all the undulations. This is also the ratio of local area $A$ to the area $A_{\mathrm{P}}$ projected onto the plane perpendicular to $N$. In the small angle approximation,

$\langle 1 / \cos \theta\rangle \approx 1+\left\langle\theta^{2} / 2\right\rangle \approx 1 /\langle\cos \theta\rangle$.

At least two previous studies $[97,98,178]$ have derived formulas that, for the regime of interest to us, reduce to

$\left\langle\theta^{2} / 2\right\rangle=\left(k T / 4 \pi K_{\mathrm{c}}\right) \ln [\pi \xi / a]$

where $k T$ is thermal energy, $K_{\mathrm{c}}$ is the bending modulus, $a$ is the mean lateral size of lipids $(\approx 8 \AA)$ and $\xi^{4}=K_{\mathrm{c}} / B . \quad B$ is the effective compression modulus which is obtained experimentally using Eq. 10 and 
measurements of $\eta_{1}$. Numerical values of these quantities are given in Table 4. (It may be of interest to note that the root mean square values of $\theta$ are of order 10 degrees.)

Table 5 shows corrected EDP values of $A$ that were obtained using both this fluctuation correction in Eq. 6 and also the new and larger values of $K_{\mathrm{A}}$ [17]. Compared to the older values of $A$ in Table 3, the new fluctuation correction increases $A$ (by $1-2 \%$ ) and the better $K_{\mathrm{A}}$ values decrease it, with a small net average increase of $0.4 \AA^{2}$ for the lipids in Table 5 .

\subsection{Corrections to $G X$ and $G X C$ results}

We first show that the GX and GXC methods are also affected by fluctuation geometry. The GX area is defined by

$A_{\mathrm{GX}}=2\left[V_{\mathrm{L}}+\left\langle n_{\mathrm{W}}\right\rangle V_{\mathrm{W}}\right] / D$,

where angular brackets denote average values. For convenience we again assume here that the bilayer is stiff relative to the water layer; the methods and notation used in Section 7.3 show that the same result is obtained without this simplifying assumption. Then, $2\left\langle n_{\mathrm{W}}\right\rangle V_{\mathrm{W}}=A\left\langle D_{\mathrm{W}}\right\rangle$ and $2 V_{\mathrm{L}}=A D_{\mathrm{B}}$, so Eq. 19 becomes

$A_{\mathrm{GX}}=A\left[D_{\mathrm{B}}+\left\langle D_{\mathrm{W}}\right\rangle\right] / D=A\langle\cos \theta\rangle$

Table 5

Adjusted areas $\left(\AA^{2}\right)$ for results in Table 3

\begin{tabular}{lllll}
\hline $\begin{array}{l}\text { Lipid } \\
\text { Temperature }\end{array}$ & $\begin{array}{l}\text { DPPC } \\
50^{\circ} \mathrm{C}\end{array}$ & $\begin{array}{l}\text { DMPC } \\
30^{\circ} \mathrm{C}\end{array}$ & $\begin{array}{l}\text { DOPC } \\
30^{\circ} \mathrm{C}\end{array}$ & $\begin{array}{c}\text { EPC } \\
30^{\circ} \mathrm{C}\end{array}$ \\
$\begin{array}{llll}\text { Method } \\
\text { GX }\end{array}$ & $72.9^{\mathrm{a}, \mathrm{b}}$ & $67.6^{\mathrm{a}, \mathrm{b}}$ & $84.4^{\mathrm{a}, \mathrm{b}}$ & $78.3^{\mathrm{a}, \mathrm{b}}$ \\
& $69.6^{\mathrm{b}}$ & $61.2^{\mathrm{a}, \mathrm{b}}$ & $76.9^{\mathrm{a}, \mathrm{b}}$ & $72.1^{\mathrm{a}, \mathrm{b}}$ \\
& & & & $66.8^{\mathrm{a}, \mathrm{b}, \mathrm{c}}$ \\
GXC & $68.9^{\mathrm{b}, \mathrm{c}}$ & $63.4^{\mathrm{a}, \mathrm{b}, \mathrm{c}}$ & $73.6^{\mathrm{a}, \mathrm{b}, \mathrm{c}}$ & $71.2^{\mathrm{a}, \mathrm{b}, \mathrm{c}}$ \\
EDP & $63.3^{\mathrm{b}, \mathrm{c}}$ & $59.5^{\mathrm{a}, \mathrm{b}, \mathrm{c}}$ & & \\
Neutron & $65.6^{\mathrm{b}, \mathrm{c}}$ & $72.5^{\mathrm{b}, \mathrm{c}}$ & $69.4^{\mathrm{b}, \mathrm{c}}$ \\
Unilamellar & $64.9^{\mathrm{a}, \mathrm{e}}$ & $60.3^{\mathrm{a}, \mathrm{e}}$ & $68.2^{\mathrm{a}, \mathrm{e}}$ & \\
\hline
\end{tabular}

The original results given in the corresponding location in Table 3 have been adjusted due to

${ }^{a}$ Temperature (see Section 7)

${ }^{\mathrm{b}}$ Fluctuation geometry (see Section 7.1)

${ }^{\mathrm{c}}$ New $K_{\mathrm{A}}[17]$

${ }^{\mathrm{d}} D_{\mathrm{C}}$ (see Section 8.1)

${ }^{\text {e}}$ Patterson truncation and $D_{\mathrm{H} 1}$ reduction (see Section 10.1). where the latter equality simply reflects what is shown in Fig. 10, namely, that the total thickness, $D_{\mathrm{B}}+D_{\mathrm{W}}$, of the local unit cell is smaller by the factor of $\cos \theta$ than the overall $D$ spacing (see Eq. 23 in Section 7.3.). This may seem counterintuitive if one imagines that the $\theta=0$ section is the reference state for non-undulating bilayers, but this is not the case. If there are no undulations, the repeat spacing is not the same $D$ but is $D_{0}=D\langle\cos \theta\rangle$ because compressing sections with maximal $\theta$ and expanding sections with $\theta=0$ costs less free energy than compressing all the sections with non-zero $\theta$, as shown in Section 7.3. Eq. 20 therefore shows that undulations alone make the apparent $A_{\mathrm{GX}}$ smaller than the true local $A$. (Note that this correction is quite independent of the earlier critique (see Section 4) of the GX method regarding the gravimetric method for obtaining $\left\langle n_{\mathrm{W}}\right\rangle$.) Table 5 shows corrected values of the literature GX results that were shown in Table 3. The correction first uses Eq. 18 with the values of $\left\langle\theta^{2} / 2\right\rangle$ in Table 4 when $P=0$ to obtain $\langle\cos \theta\rangle$ in Eq. 17, which is then used in Eq. 20 to obtain $A$ from the apparent $A_{\mathrm{GX}}$. Finally, the results are adjusted to common temperatures using the area thermal expansivity $\alpha$.

Table 5 also shows corrected values of the literature GXC results shown in Table 3. The literature values had been extrapolated to $P=0$ from $P=10$ atm using the apparent or assumed values of $K_{\mathrm{A}}$ [1]. This extrapolation was first undone using Eq. 2 in which values of $D_{\mathrm{W}}$ were obtained using results given in Table VIII in [1]. The undulation correction in Eq. 20 was then applied using results in Table 4 for $P=10 \mathrm{~atm}$, which was the value used in the GXC method [1]. Then, the result was re-extrapolated to $P=0$ using the new experimental values of $K_{\mathrm{A}}$ for DOPC and DMPC [17]. For DPPC and EPC a value $K_{\mathrm{A}}=250 \mathrm{dyn} / \mathrm{cm}$ was estimated using the arguments that the value for EPC should be similar to DOPC and that the effect of longer chains in DPPC compared to DMPC should be somewhat compensated by the higher temperature. (Evan Evans has recently informed us that his group has obtained $K_{\mathrm{A}}=248 \pm 20 \mathrm{dyn} / \mathrm{cm}$ and $K_{\mathrm{c}}=0.58 \pm 0.04 \times 10^{-12}$ erg for EPC and $K_{\mathrm{A}}=231 \pm 20 \mathrm{dyn} / \mathrm{cm}$ for DPPC.) Finally, the modification to common temperatures was made as above. Comparison of Table 5 to Table 3 shows that the average increase in $A$ from all these 
corrections is $2.2 \AA^{2}$ for the GX results and $1.4 \AA^{2}$ for the GXC results. The increase in the GX values of $A$ was larger because there was no compressibility adjustment, which contributed a decrease to the GXC value of $A$. The temperature adjustment increased the average area by $0.8 \AA^{2}$. This accounts for part of the reason that the adjusted increases in the GXC values were larger than for the EDP values. The other reasons are that the EDP values were obtained at higher $P$ where the fluctuation correction is smaller and many of the best estimates of $K_{\mathrm{A}}$ had previously been smaller.

\subsection{Bilayer deformability and fluctuations}

We return here to the assumptions made in the last two subsections. It is necessary to establish notation with regard to variations in the bilayer thickness $D_{\mathrm{B}}$, the water layer thickness $D_{\mathrm{W}}$, and the local area $A$ when there are undulations involving sections with varying slope $\theta$. Of course, for any section there are fluctuations around the mean values, but the notation need not emphasize this averaging. The important distinction is that the mean values vary with $\theta$, according to

$$
\begin{aligned}
& D_{\mathrm{B}}(\theta)=D_{\mathrm{B}}+\Delta D_{\mathrm{B}}(\theta), D_{\mathrm{W}}(\theta)= \\
& \quad D_{\mathrm{W}}+\Delta D_{\mathrm{W}}(\theta), A(\theta)=A+\Delta A(\theta)
\end{aligned}
$$

where $D_{\mathrm{B}}, D_{\mathrm{W}}$ and $A$ are defined to be the mean values averaged over all $\theta$, so the averages $\left\langle\Delta D_{\mathrm{B}}(\theta)\right\rangle$, $\left\langle\Delta D_{\mathrm{W}}(\theta)\right\rangle$ and $\langle\Delta A(\theta)\rangle$ over $\theta$ are identically zero. There are two constraints on the mutual variations. The first is the volume constraint, $A(\theta) D_{\mathrm{B}}(\theta)=V_{\mathrm{L}}$. Ignoring second-order variations, this yields

$\Delta A / A=-\Delta D_{\mathrm{B}} / D_{\mathrm{B}}$,

where, for convenience, the explicit dependence upon $\theta$ will now be omitted from the notation for the $\Delta$ quantities. The second constraint is the geometric stacking constraint that $D_{\mathrm{B}}(\theta)+D_{\mathrm{W}}(\theta)=D \cos \theta$, so

$D_{\mathrm{B}}+D_{\mathrm{W}}=D\langle\cos \theta\rangle, \Delta D_{\mathrm{B}}+\Delta D_{\mathrm{W}}=D \Delta \cos \theta$.

The appropriate calculation minimizes the free energy $F$ with respect to these coupled variations. The part of the free energy associated with changes in the bilayer is, for each section with tilt $\theta$,

$F_{\mathrm{A}}(\theta)=\left(K_{\mathrm{A}} / 2\right)\left(A+\Delta A-A_{0}\right)^{2} /(A+\Delta A)$

where $A_{0}$ is defined to be the mean area when $P=0$. The part of the free energy $F_{\mathrm{I}}$ associated with changes in the water thickness involves all the different interlamellar interactions between bilayers. We consider only the strong hydration force since the other forces are only important very near full hydration where this calculation is not important since $D_{\mathrm{B}}$ is practically constant near full hydration. Then,

$F_{\mathrm{I}}(\theta)=P \lambda_{\mathrm{h}} A(\theta) \exp \left(-\Delta D_{\mathrm{W}} / \lambda_{\mathrm{h}}\right)$.

There is also a part of the free energy $F_{\mathrm{P}}$ that comes from the osmotic pressure,

$F_{\mathrm{P}}=P A(\theta) D_{\mathrm{W}}(\theta)$.

In the next step the free energy, $F=F_{\mathrm{A}}+F_{\mathrm{I}}+F_{\mathrm{P}}$, is expanded in powers of the $\Delta$ quantities. Averaging over all $\theta$ makes all first order terms vanish. After using Eq. 22 to eliminate $\Delta A$ terms in favor of $\Delta D_{\mathrm{B}}$, we then have

$$
\begin{gathered}
\langle F\rangle=F_{0}+\left[\left(P A / \lambda_{\mathrm{h}}\right)\left\langle\left(\Delta D_{\mathrm{W}}\right)^{2}\right\rangle+\right. \\
\left.\left(K_{\mathrm{A}} A / D_{\mathrm{B}}^{2}\right)\left\langle\left(\Delta D_{\mathrm{B}}\right)^{2}\right\rangle\right] / 2
\end{gathered}
$$

where $F_{0}$ is the free energy for bilayers with the mean values in Eq. 21, the second term on the right hand side is the excess free energy of water layer deformation and the last term is the excess free energy of bilayer deformation. Both the latter terms increase with increasing undulations, which would seem to predict that undulations should be absent. However, this $F$ should be supplemented by a strictly entropic undulation free energy contribution which is independent of any of the variables in Eq. $21[47,84]$.

Given that there are undulations, Eq. 27 enables us to obtain the relative sizes of the bilayer deformation and the water layer deformation by minimizing $\langle F\rangle$ subject to the second constraint in Eq. 23. This requires $\Delta D_{\mathrm{W}} / \Delta D_{\mathrm{B}}$ to be a constant for all $\theta$ and the value of this ratio that minimizes $\left\langle F-F_{0}\right\rangle$ is

$\Delta D_{\mathrm{W}} / \Delta D_{\mathrm{B}}=\lambda_{\mathrm{h}} K_{\mathrm{A}} / P D_{\mathrm{B}}^{2}$.

This ratio is about 2.5 when $P=10$ atm, indicating that the water layer is still more deformable than the bilayer. 
Perhaps the most important result from Eq. 27 concerns the $F_{0}$ term which only depends upon the average values in Eq. 21. If one suppresses fluctuations, $\langle F\rangle=F_{0}$ identifies $F_{0}$ with the free energy of non-fluctuating bilayers and this identifies $D_{\mathrm{B}}, D_{\mathrm{W}}$ and $A$ with the values they would assume in nonfluctuating bilayers. Therefore, the partitioning of the deformations with varying $\theta$ into water versus bilayer is irrelevant to average values of $D_{\mathrm{B}}, D_{\mathrm{W}}$ and $A$. The important exception for subsection 7.2 is that $D$ increases with increasing fluctuations as shown by Eq. 23. The effects of geometric fluctuations therefore depend only upon $\left\langle\theta^{2}\right\rangle$ as derived in Sections 7.1 and 7.2.

\section{Neutron diffraction}

Neutron diffraction is at a disadvantage to X-ray diffraction in that neutron beams are much weaker and there are fewer sources of neutrons. Nevertheless, neutron diffraction is quite valuable as indicated in the following two subsections.

\subsection{Specific deuteration}

Neutrons have one great advantage over X-rays because deuteration dramatically changes the scattering of neutrons. Specific deuteration of component parts of the lipid, such as a selected methylene, therefore provides a localized contrast agent that leaves the system physically and chemically nearly equivalent. In their classic study of DPPC, Büldt et al. [10] obtained difference form factors by subtracting nondeuterated form factors from specifically deuterated form factors. The distribution function for the specifically deuterated group corresponds to these difference form factors. Büldt et al. [10] fit the form factors of a model Gaussian distribution function, with mean position $z_{\mathrm{g}}$ of a group $g$ and a 1/e half-width $v_{\mathrm{g}}$, to the difference form factors. This choice replaces errors due to Fourier truncation with errors due to non-Gaussian distributions. Perhaps this latter approximation accounts for the apparently anomalous result that the best obtained distance between the fourth and fifth methylene groups $(1.7 \AA)$ in their most hydrated samples is larger than the $\mathrm{C}-\mathrm{C}$ bond length $(1.54 \AA)$. However, the quoted errors in each of the positions are quite large $(1.5 \AA)$, so distances between two close groups were not well determined. The most hydrated DPPC samples contained 25\% water [10]; this corresponds to $n_{\mathrm{W}}=13.6$ if one makes the standard gravimetric assumption. This water content sufficed to give nearly the same $D$ as fully hydrated gel phase DPPC. Although it was worrying that for the fully hydrated $L_{\alpha}$ phase their $D=54.1 \AA$ fell well short of fully hydrated $D=67 \AA$, it was later suggested that this was not a concern because X-ray form factors seemed to indicate little change in bilayer thickness over this range of hydration [3].

Büldt et al. [10] reported $A=57 \AA^{2}$ for the $L_{\alpha}$ phase of DPPC by extrapolating from the gel phase in a way that is conceptually similar to the X-ray EDP method described in Section 5. They only used the measured volume change at the main phase transition instead of the volume change from $20^{\circ} \mathrm{C}$ to $50^{\circ} \mathrm{C}$; correcting this raises their $A$ by about $3 \%$. Another improvable assumption was that the lipid occupied all the volume up to $z_{\beta}$ for the position of the $\beta$ carbon on the choline moiety of the headgroup, but as $A$ expands from the gel to the $L_{\alpha}$ phase, more water enters into the headgroup region (see Fig. 2a). Two alternative ways to obtain $A$ from the neutron data were suggested [3]. One method is very similar to the extrapolation method used for Xray studies in Section 5 . The other method uses $z_{4}$ and $z_{5}$ for the fourth and fifth carbons in the fluid phase and compares hydrocarbon chain volumes; this method also requires knowing methylene and terminal methyl volumes. Results for both methods are in substantial agreement, giving a best value of $A=63 \AA^{2}$, although the errors in $A$ that are propagated from the quoted errors in mean positions are quite large $\left(\approx 7 \AA^{2}\right)$. Since then, the necessity of a compressibility correction has been recognized and a geometric fluctuation correction is now proposed in Section 7. These adjustments have also been made to the value that we give in Table 5 as a replacement for the original value shown in Table 3 .

The results of Büldt et al. [10] have been an important guide for estimating the headgroup thickness $D_{\mathrm{H}}{ }^{\prime}$ and hence the steric membrane thickness $D_{\mathrm{B}}{ }^{\prime}$ [26,66] (compare Fig. 2a,c). Their results also provide, in principle, a direct measure of local fluctuations of molecular groups within the bilayer. The 1/e 
half-widths $v_{\mathrm{g}}$ were of order $3 \AA$ for most groups although with smaller $v_{\mathrm{gly}}$ for the glycerol group. However, half-widths could only be obtained for samples that had less than $10 \%$ water because not enough orders of diffraction could be obtained to fit the $v_{\mathrm{g}}$ parameter for their more hydrated samples with $25 \%$ water.

\subsection{Joint refinement of neutron and X-ray data}

Without the benefit of costly specific deuteration, neutron diffraction is not to be preferred to X-ray diffraction. Of course, it does provide a different experimental window on lipid bilayer structure, and a basic tenet in biological sciences is that different perspectives have cumulative value. Wiener and White, however, have shown for lipid bilayers that the synergy between X-ray and neutron diffraction can be much better than just comparing results obtained independently. Their approach was to fit both Xray form factors and neutron form factors (usually called scattering lengths) simultaneously to a model of lipid molecular components and water. This approach essentially doubles the amount of data that can be used to determine a more refined structure. Joint refinement requires that a model be chosen and they worked exclusively with Gaussian distribution functions. The errors generated by this choice have been studied using simulations [21,22]. Even with combined neutron and X-ray data, there are too many different component groups for one Gaussian each. It was therefore necessary to combine the chain methylenes into a smaller number of distributions and Wiener and White chose three Gaussians for this purpose. An alternative, used in X-ray analysis [39], would be to model the methylene density as a constant within the hydrocarbon region, with fuzzy edges near $D_{\mathrm{C}}$ and a functional form suggested from simulations, as in Fig. 2a (H.I. Petrache, personal communication). Another alternative used volumetric constraints and this resulted in a better fit to the $\mathrm{X}$-ray and neutron data [22] than in the original work.

In a series of papers Wiener and White $[19,25,43]$ thoroughly studied fluid phase DOPC at $66 \%$ relative humidity and obtained molecular component distribution functions like those shown in Fig. 2a. However, this is a fairly dry sample, with $n_{\mathrm{W}}=5.4$ [44].
Simulations indicate that $n_{\mathrm{W}}=11-13$ is necessary to complete the inner hydration shell of the lecithin headgroup [99,101]. The greatest concern is that the reported value of $A=59.3 \AA^{2}$ is so much smaller than the other values in Table 3. Of course, $66 \%$ RH corresponds to a large equivalent osmotic pressure, $P=570 \mathrm{~atm}$, so Eq. 2 should be used to estimate the fully hydrated $A_{0}$. However, using $K_{\mathrm{A}}=265$ dyn/cm [17], $P=570$ atm, and $D_{\mathrm{W}}=2.5 \AA$, Eq. 2 still predicts only $A_{\mathrm{O}}=62.5 \AA^{2}$. For the value shown in Table 5 under DOPC/neutron, we have also adjusted for temperature differences, but no geometric fluctuation correction was made since undulations are suppressed at such high osmotic pressures. It therefore seems that straightforward corrections and adjustments do not suffice to extrapolate $A$ for DOPC at $66 \%$ relative humidity to a value appropriate for full hydration. This agrees with a study of Hristova and White which showed that substantial, abrupt changes take place when $P$ increases into the range near $100 \mathrm{~atm}$ where $n_{\mathrm{W}}$ is about 12 [100]. This discouraging outcome for DOPC is a warning that the compressibility extrapolation of the GXC method may not work to obtain fully hydrated structure, especially when the reference state is too dry. Nevertheless, this DOPC study [25] illustrates that the combination of neutron and X-ray diffraction should be a powerful tool for future structural studies of lipid bilayers.

\section{NMR}

\subsection{Order parameter method}

As shown in Fig. 1, the NMR deuterated methylene order parameters $S_{\mathrm{CD}}$ have provided as wide a variety of values of $A$ for DPPC as have diffraction methods. A striking difference for NMR is that there is little disagreement in values of the basic data for the order parameters. The disparities arise in the way that the chain length and $A$ are derived from the order parameters. Three separate binary choices have been elucidated and a particular set of choices was advanced that yielded $A_{\mathrm{DPPC}}^{\mathrm{F}}=62 \AA^{2}$ [102]. One formula that many, but not all, workers had agreed upon relates the chain travel distance along the normal to the bilayer $D_{n}$ to order param- 
eters $S_{n}$ by

$\left\langle D_{n}\right\rangle / D_{\mathrm{M}}=\left(1-2\left\langle S_{n}\right\rangle\right) / 2$

where $D_{\mathrm{M}}=1.27 \AA$ is the maximum travel per methylene for all-trans chains oriented perpendicular to bilayer. However, this formula did not fit data from two subsequent molecular dynamics simulations [2,103]. A better formula that was inspired by the simulations and that fits them well $[2,103,104]$ is

$\frac{\left\langle D_{n}\right\rangle}{D_{\mathrm{M}}}=\frac{1}{2}\left(1+\sqrt{\frac{-8\left\langle S_{n}\right\rangle-1}{3}}\right)$.

Addition of $\left\langle D_{n}\right\rangle$ for all carbons $n$ then yields the average hydrocarbon chain length $L_{\mathrm{C}}$.

If $L_{\mathrm{C}}$ were shorter than $D_{\mathrm{C}}$, this would have explained [102] why modern NMR values [16] were on the high end in Fig. 1 because $L_{C}$ was used instead of $D_{\mathrm{C}}$ in the basic average volumetric formula [16]

$A=V_{\mathrm{C}} / D_{\mathrm{C}}$.

However, one of the simulations [2] indicated no significant difference in the length of the average hydrocarbon chain compared to the hydrocarbon thickness $D_{\mathrm{C}}$. Also, despite the significant differences between Eq. 29 ad 30 for individual methylenes $n$, the sums over $n$ give values of $L_{\mathrm{C}}$ that are very nearly the same, so this innovation does not change the previous results of Brown's group [16]. Furthermore, the result for $A$ from Eq. 31 nicely reproduces the actual $A$ in the simulation [2]. Unfortunately, when applied to real data for DMPC [63], Eq. 31 gives a value of $A=65.4 \AA^{2}$ [2], considerably larger than the best diffraction results. In the next three paragraphs we will mention three proposals that might reconcile this difference.

One way to reconcile the $A$ from nmr with diffraction results is to use the value of the order parameters only in the plateau region, as advocated earlier [102]. This choice implies that $D_{\mathrm{C}}$ is larger than $L_{\mathrm{C}}$. This method is suspect if there is no spatial region that is only occupied by methylenes [2]. A recent paper reconciles the nmr results with diffraction results by using this choice of plateau order parameters together with a more accurate way to calculate averages [108]. Simulations could further address the validity of this proposal.

The nmr values obtained using Eq. 31 have not been corrected for geometric fluctuations discussed in Section 7.1. NMR is also affected by this correction because projected chain travel along $N$ is less, by a factor of $\cos \theta$, than the travel along the molecular axis, as has been previously pointed out [98]. Using the data from Table 4, the correction to $A$ involves division by $1+\left\langle\theta^{2} / 2\right\rangle$. This reduces the above NMR value of $A$ for DMPC from $65.4 \AA^{2}$ [2] to $64.0 \AA^{2}$ and the value for DPPC from $70.7 \AA^{2}$ [16] to 69.7 $\AA^{2}$. These values are still disturbingly high. Koenig et al. [63] did not use their NMR values for $A$, but instead used NMR to provide $K_{\mathrm{A}}$ to extrapolate to full hydration using the GXC method. As shown in Table 5, their result and the independent EDP result agree that $A_{\text {DMPC }}^{F}$ is less than $60 \AA^{2}$. The NMR result for DPPC is also even higher than the GXC result which in turn is likely to be somewhat too high due to residual defect water. It is, however, intriguing that if the fluctuations were large enough, then the NMR value would be decreased and the EDP value would be increased until they became equal. This would require $K_{\mathrm{c}}$ values in the range of $0.1-0.2 \times 10^{-12}$ erg which is in the same range as given by Niggeman et al. [109] for DOPC using the video microscopy method, and deHaas et al. [110] using shear flow deformation. This is much lower than the DOPC value of $0.8 \times 10^{-12}$ erg obtained by the aspiration pipette method [17]. It might be noted that the video microscopy method does not give consistently smaller values; for DMPC it gave $1.1-1.3 \times 10^{-12}$ erg $[95,111]$ compared to $K_{\mathrm{c}}=$ $0.56 \times 10^{-12} \mathrm{erg}$ obtained from the aspiration pipette method [17]. The determination of $K_{\mathrm{c}}$ is clearly a central issue for this proposal.

Since the $S_{\mathrm{CD}}$ method works so well for simulations, the third reconciliation proposal is that the experimental NMR order parameters may be too low because of other motions and time scale issues that might reduce the magnitude of $S_{\mathrm{CD}}$ compared to static, geometric values $[105,106]$. If so, a quantitative analysis would be required to obtain $A$ independently from $S_{\mathrm{CD}}$ order parameter measurements.

We are unwilling to firmly endorse any of the three preceding proposals and therefore feel that there is still considerable uncertainty in obtaining $A$ using the NMR order parameter method. It may also be noted that the NMR value of $K_{\mathrm{A}}=140 \mathrm{dyn} / \mathrm{cm}$ for DMPC [63] agreed very well with the older apparent value 
[94] but both are considerably smaller than the newer true value shown in Table 4. However, the geometric fluctuation correction was also not applied to the NMR data. Although the correction is somewhat different in form and the numbers are not in perfect agreement, the effect of including this correction is to increase the NMR value of $K_{\mathrm{A}}$ closer to the new true values [17].

\subsection{Magic angle spinning (MAS)}

Recently, a different way to use NMR to obtain $A$ has been elucidated [112]. This is based on magic angle spinning (MAS) measurements of the water proton signal, which has a different chemical shift depending upon whether the water is in bulk or whether it interacts with bilayers [113]. The idea is to obtain values of $n_{\mathrm{W}}$ which can then be used in Eq. 1 to obtain $A$. The usual complication is that interacting water is not located just between the bilayers in MLVs, but also includes water in defect regions between MLVs (see Fig. 3) [112]. By increasing the frequency of centrifugation an effective, non-uniform, osmotic pressure is applied to the MLVs which removes water from the defects and also from between the bilayers. This latter water loss is provided from X-ray studies. This lost interlamellar water is then added back to the total interacting water and the result is extrapolated as a function of spinning frequencies to the limit of high spinning frequencies where the defect water is minimized. The extrapolation yields a value of $n_{\mathrm{W}}$ for fully hydrated DOPC which is in good agreement with that obtained by the EDP method [38].

\section{Single bilayers}

\subsection{Unilamellar vesicles}

Unilamellar vesicles are more biologically appealing models of lipid bilayers than MLVs. One obvious concern with MLVs is that the interaction between different bilayers, which is absent in unilamellar vesicles and most membranes, might alter the bilayer structure. Although this is a concern for low levels of hydration, the discussion at the end of Section 6.3 shows that the interactions between bilayers are too small to affect fully hydrated bilayer structure when the water layer exceeds $D_{\mathrm{W}^{\prime}}>10 \AA$ and $n_{\mathrm{W}}-n_{\mathrm{W}}{ }^{\prime}>10$, so this is not a compelling reason to prefer the study of unilamellar lecithin bilayers in preference to MLVs. The disadvantage of unilamellar vesicles compared to MLVs is, of course, the small concentration of bilayers that can be studied, so the intensity is very small and signal-to-noise is poor even for modest values of $q$ corresponding to $h=3$ orders. Concentrating the sample necessarily leads to correlation in positions between bilayers and to the necessity of including poorly determined and rather diffuse $S(q)$ structure factors.

Lewis and Engelman studied a series of unilamellar lecithins using Patterson function analysis to obtain $D_{\mathrm{HH}}$ [12]. Of course, this analysis is also subject to truncation error due to limited data in $q$; using either a model electron density function or the simulation data in Fig. 2a we estimate that the Patterson analysis would predict $D_{\mathrm{HH}}$ to be about $0.8-1.0 \AA$ smaller than the actual $D_{\mathrm{HH}}$ using the same $q$ range of the data obtained by Lewis and Engelman. To obtain values of $A$ Lewis and Engleman then assumed that the distance $\left(D_{\mathrm{HH}} / 2\right)-D_{\mathrm{C}}$, which we call $D_{\mathrm{H} 1}$, was $5.5 \AA$ and then volumetric data were used to obtain $A$ with results shown in Table 3. This value of $D_{\mathrm{H} 1}$ is what one measures for the distance between the phosphate and the average hydrocarbon chain boundary using a molecular model of a gel phase lipid with the glycerol backbone aligned along the bilayer normal. Molecular tilt and conformational disorder reduces this value to about $5.2 \AA$ in the simulation shown in Fig. 2a. We have used this latter value of $D_{\mathrm{H} 1}$ and the above correction to $D_{\mathrm{HH}}$ and a temperature adjustment (see Section 7) to obtain the values shown in Table 5 in the row labelled 'unilamellar'.

Unilamellar vesicles have also been studied more recently by neutron scattering [114-116]. Because of the high contrast between protonated lipid and deuterated water, the first order model consists of a bilayer part with a different scattering length from the water, with only one thickness parameter. Because signal-to-noise limits the data to $q<0.2 \AA^{-1}$, Mason et al. [116] note that more refined models make little improvement and accurate measurement of absolute bilayer thickness is precluded. However, relative changes can be readily detected, which is 
useful for an application that is discussed in Section 11.

\subsection{Bilayers on a solid substrate}

It is useful for many applications to support a single lipid bilayer on a solid substrate and then reflectometry is the appropriate scattering technique. In order to provide a stable bilayer, a recent study prepared hybrid bilayers composed of an alkanethiol monolayer attached to a gold surface with a DPPC monolayer facing water [117]. Reflectometry data to $q=0.25 \AA^{-1}$ enabled determination of changes in thickness between gel and $L_{\alpha}$ phase DPPC that are consistent with those obtained by the EDP method and the deduced values of hydrocarbon thickness are only about $1.5 \AA$ smaller. Of course, the hybrid nature of the bilayer and the interactions with the substrate make such model systems unsuited for primary bilayer structure determination and [117] emphasizes the value of primary determinations to ensure the validity of using these bilayers as model systems for applications.

\subsection{Monolayers}

Monolayers at an air/water interface on a Langmuir trough are attractive because it is much easier to measure $A$ directly than in bilayers [118]. However, $A$ varies with the applied surface monolayer pressure $\pi_{\mathrm{m}}$, so prediction of $A$ for bilayers using monolayers requires knowing what $\pi_{\mathrm{m}}$ to apply. Marsh has advocated that $\pi_{\mathrm{m}}$ should be in the range 30-35 dyn/cm [119], but the main transition temperature $T_{\mathrm{M}}$ for DPPC then occurs some $5^{\circ} \mathrm{C}$ too low compared to bilayers. Other authors have suggested that $\pi_{\mathrm{m}}$ should be close to $50 \mathrm{dyn} / \mathrm{cm}$ [120-122]; this gives the correct $T_{\mathrm{M}}$, but $A$ for DPPC monolayers at $\pi_{\mathrm{m}}=50 \mathrm{dyn} / \mathrm{cm}$ and $T=50^{\circ} \mathrm{C}$ is less than our best bilayer value of $A=64 \AA^{2}$ and only achieves this latter value at $T=50^{\circ} \mathrm{C}$ when $\pi_{\mathrm{m}}$ is near $28 \mathrm{dyn} / \mathrm{cm}$ [123]. Using monolayers to predict bilayer properties seems reasonable if bilayers can be treated as two back-to-back monolayers interacting non-specifically as two slabs. This latter assumption has been challenged $[120,124,125,180]$. Indeed, the degree of overlap of simulated distribution functions of the terminal methyls from the opposing monolayers in the $L_{\alpha}$ phase suggest that there must be specific intermonolayer interactions in this phase (Scott Feller, private communication). In contrast, the interaction between the monolayers in gel phase bilayers would be expected to be more slab-like, although even for this phase some specific interaction must remain because the chains in both monolayers are tilted in parallel [4], as will be discussed in Section 11.1. The tilt angle $\theta_{\mathrm{t}}$ and $A$ of DPPC monolayers at $20^{\circ} \mathrm{C}$ also vary with $\pi_{\mathrm{m}}$. The values of $\theta_{\mathrm{t}}$ that are closest to those of gel phase DPPC bilayers occur when $\pi_{\mathrm{m}}$ slightly exceeds $42 \mathrm{dyn} / \mathrm{cm}$ [126]. It therefore seems that the $L_{\alpha}$ phase, that has the strongest specific interactions between monolayers, requires $\pi_{\mathrm{m}}=28 \mathrm{dyn} / \mathrm{cm}$ and this is raised to somewhat greater than $42 \mathrm{dyn} / \mathrm{cm}$ for the gel phase that has weaker specific intermonolayer interactions. This progression of $\pi_{\mathrm{m}}$ values supports the theoretical estimate that $\pi_{\mathrm{m}}$ would be 50 $\mathrm{dyn} / \mathrm{cm}$ if there were no specific interactions between the monolayers in a bilayer. However, it now seems clear that there are such interactions that are still not clearly defined. We suggest that, instead of trying to obtain correspondence between monolayers and bilayers and using one system to elucidate the other, future effort should probably focus on the differences between them as determined by independent measurements and simulations.

\section{Chain ordered phases}

\subsection{Gel phase}

The thermodynamic phase of DPPC that is best characterized is the so-called gel or $L_{\beta}{ }^{\prime}$ phase because wide-angle scattering yields additional information that allows $A$ to be determined directly. The distinctive wide-angle patterns show that the hydrocarbon chains in this phase are parallel to each other in a nearly hexagonal array. If the chains were oriented along the bilayer normal, then indexing the wide-angle reflections to determine the inplane unit cell gives $A$ directly. The problem of determining the structure of the $L_{\beta}{ }^{\prime}$ phase is made more challenging by the tilting of the hydrocarbon chains. Chain tilt $\left(\theta_{\mathrm{t}}\right)$ gives rise to two additional order parameters. The first is the relative orientation $\phi_{\mathrm{t}}$ of the tilt $\theta_{\mathrm{t}}$ with the two special cases being tilt 
towards nearest neighbors (nn) or tilt towards next nearest neighbors (nnn). Safinya and co-workers showed that both these phases, as well as a phase with intermediate tilt direction, exist for different degrees of dehydration in free standing films of DMPC $[52,85]$. Both phases have also been found in DPPC [127]. However, for fully hydrated DPPC or DMPC, the relative orientation is always (nn). (A still unresolved issue is that the extrapolation of the free standing film data [52,85] to full hydration predicts the wrong (nnn) phase.) For the (nn) phase there is a (20) reflection with only an in-plane $q_{\mathrm{r}}$ component and a (11) reflection which also has a $q_{\mathrm{z}}$ component whose relative size is related to the tilt angle $\theta_{\mathrm{t}}$. Using fully hydrated oriented samples enabled direct measurement of all $q$ components for all wide-angle reflections, and then a tilt angle of $32^{\circ}$ was obtained $[41,53]$. Combining this tilt angle with polarized attenuated total reflection infrared data also allowed estimates for a different order parameter that relates to rotation of the hydrocarbon chains about the longchain axes [128].

Although it is usually more ambiguous to determine patterns from powder samples, many workers had, in fact, deduced the (nn) pattern for the gel phase of DPPC from powder samples, but quantitative measurement of the tilt angle $\theta_{\mathrm{t}}$ was only obtained indirectly via the GX method [42]. However, there are additional, small features in the wide-angle powder pattern that allowed independent evaluation of $\theta_{t}$ for powder samples of DPPC. Furthermore, because the powder data had better statistics and fewer artifacts like mosaicity, a more detailed model could be fit [4]. Another older result that was confirmed is that the chains in one monolayer of the bilayer are parallel to the chains in the other monolayer. What brings about this specific interaction between the two monolayers is still not well understood. One hypothesis is a slight mini-interdigitation between the ends of the chains because the $s n-1$ chain penetrates more deeply into the bilayer. A test of this hypothesis would be to examine the gel phase of MPPC, for which the penetration should be more nearly equal and therefore have minimal interdigitation. Remarkably, however, MPPC has no gel phase at all [129], going directly from subgel to ripple to fluid phase [130]. It may also be noted that molecular dynamics simulations have tended to obtain pleated chain packing structures and have only recently succeeded in obtaining the parallel chain structure of the gel phase [131], suggesting that the interactions that bring this pattern about are weak and subtle. A new result from the fitting to the powder data showed that the parallel chains from each monolayer are offset relative to each other rather than being perfectly collinear [4].

There is still an unresolved dichotomy regarding fluctuations in the gel phase. While the $(2,0)$ wideangle scattering peak is very narrow, corresponding to in-plane correlation lengths of $2900 \AA$, the total diffuse scattering intensity in the wide-angle region is even larger than the intensity under the peaks [4]. This requires a great deal of fluctuational disorder in the range $(4-10 \AA)$ of intermolecular spacings within the bilayer. One hypothesis to account for this dichotomy is that, while the scattering peaks come from well packed chains, the diffuse scattering is primarily due to the disordered head groups which scatter more strongly than the chains due to their higher electron density contrast with the solvent. However, closer analysis suggested that the chains must have considerable disorder as well $[4,128]$.

Temperature and chain length dependence have been rather completely studied for the gel phase [72]. The results are quite regular for chain lengths up to $n=20$ carbons. The picture that emerges is that there is a steric interaction between headgroups that maintains $A$ nearly constant with only a small area thermal expansivity $\alpha_{\mathrm{A}} \approx 0.0003 /{ }^{\circ} \mathrm{C}$, about ten times less than in the $L_{\alpha}$ phase. As has been known for a long time, the cause of tilt of the hydrocarbon chains arises because headgroups have a natural area $A$ that is greater than twice the natural packing area $2 A_{\mathrm{c}}$ of parallel chains (two per lipid). This frustration is relieved by chain tilt given by $\cos \theta_{\mathrm{t}}=2 A_{\mathrm{c}} / A$ $[132,133]$. We now know that the chain area $A_{\mathrm{c}}$ increases much more rapidly with $T$ than the head area $A$, so $\theta_{\mathrm{t}}$ decreases with $T$. Also, $\theta_{\mathrm{t}}$ is larger for longer chain lengths because they have larger attractive van der Waals interactions which decrease $A_{\mathrm{c}}$ [72].

For chains longer than $n=20$ carbons, new gel phase structures develop, both at low temperature and near the chain melting temperature $T_{\mathrm{M}}$, as seen in both X-ray diffraction [134] and IR and DSC [135]. It appears that the new low $T$ phase is not a traditional subgel phase. The interpretation is that it 
has untilted chains which, when compared to the usual gel phase, IR shows are more ordered and Xray shows have opposite hexagonal symmetry breaking. The new high $T$ phase appears to have hexagonal chain packing, like the ripple phase, but there is no direct evidence that it is a ripple phase.

Different kinds of gel phases have been found in a variety of lipid bilayers. One class of examples includes several varieties of phases in which the ordered hydrocarbon chains are interdigitated [136], due to the effect of solvent [137], mismatched chains [138-141] or ether [142]. There are also various classes of lipids that have interesting ordered phase behavior. For example, there are many glycosphingolipids that have chain ordered phases. The thermodynamic behavior of these phases is characterized by hysteresis, so thermal protocols must be carefully considered when characterizing the three distinct phases that were recently seen in the ceramide lipid C16:0-LacCer [143].

\subsection{Ripple phase}

Most lipids have more low temperature chain ordered phases than just the gel phase. The most striking of these is the ripple phase, which occurs just

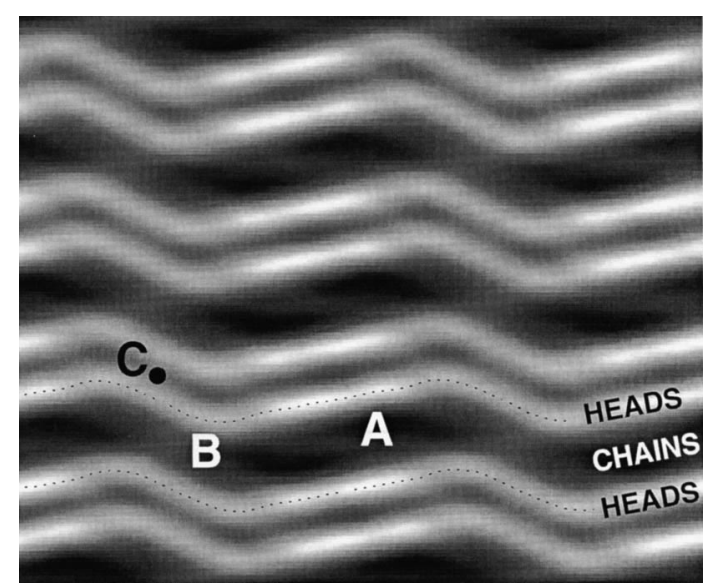

Fig. 11. Electron density map obtained using X-ray phases from [147] and intensity data from [146] for the ripple thermodynamic phase of DMPC with $25 \%$ water $\left(n_{\mathrm{W}}=13\right)$ at $18^{\circ} \mathrm{C}$. The rippling repeat period is $142 \AA$ (length of unit cell) and the lamellar repeat is $58 \AA$ (height of unit cell). The profiles show a major $M$ side (across A) that has the same thickness as the gel phase and a thinner minor $m$ side (across B). The presence of a thin water layer between bilayers (across C) indicates complete inner shell hydration of the headgroups. below the main transition in the lecithins with saturated chains. This phase has low angle diffraction peaks requiring two indices $(h, k)$ [13] where the period corresponding to $h$ is the usual lamellar spacing and the $k$ index corresponds to a repeat distance $\lambda_{\text {r }}$ $(\approx 140 \AA$ ) in the plane of the bilayer (Fig. 11). Obtaining the additional $k$ index is straightforward in the diffraction patterns of oriented samples [144] and can also be done in high instrumental resolution powder patterns from MLVs [146]. In contrast, even identifying the ripple phase can be somewhat uncertain from low resolution powder patterns for the untrained eye because the $(h, k)$ peaks for different $k$ are not resolved, although the wide-angle pattern helps identification because it is clearly different from the (nn) gel phase [46,145].

Obtaining the electron density profile for the ripple phase requires phasing the reflections. The best ripple phase data for this purpose included 26 reflections for powder samples of nearly fully hydrated DMPC with $25 \%$ water [146], but phasing these data took another eight years [147]. The electron density profile clearly shows rippling of a sawtooth variety, with a longer major $\mathrm{M}$ side that has the same thickness as gel phase DMPC and a shorter minor $\mathrm{m}$ side that is thinner (see Fig. 11). However, even with the detail that is provided by the EDP, it is still not known how the hydrocarbon chains are oriented in the bilayer. One possibility is that the major $\mathrm{M}$ side is like the gel phase and the minor $\mathrm{m}$ side is disordered like the $L_{\alpha}$ phase. A recent specific suggestion [148] has all the hydrocarbon chains ordered, but the suggested geometry has the chains perpendicular to the $M$ side which would make that side thicker than the gel phase bilayer in which the chains are tilted. More wide-angle diffraction studies will be required to determine the detailed molecular structure.

In the case of DPPC the ripple phase is complicated by the occurrence of two quite different diffraction patterns depending upon whether the sample is cooled from the $L_{\alpha}$ phase or heated from the gel phase. Powder diffraction patterns for the cooling phase were controversial, but recent use of fully hydrated aligned samples [144] proves that the indexing of Hatta's group [149] was correct. The ripple phase formed upon heating is the usual ripple phase, but the phase formed upon cooling consists of a mixture of the usual phase and a longer ripple phase. The 
proportion of the two phases depends delicately upon rate of cooling [144,150]. Although it is not proven from diffraction because no electron density profile is yet available, it is likely that the long ripple consists of an MmmM repeat pattern, in contrast to the $\mathrm{Mm}$ repeat pattern of the short ripple. Diffraction evidence includes the robust presence of a rectangular unit cell, required by the symmetry of the MmmM pattern, and the ratio of nearly a factor of two in ripple lengths. Also, freeze fracture electron microscopy indicates the $\mathrm{MmmM}$ pattern for the long ripples [151].

\subsection{Subgel phase}

The most studied subgel phase is for DPPC. Careful equilibrium studies show that the equilibrium transition temperature between subgel and gel phases is $T_{\mathrm{S}}=14.5^{\circ} \mathrm{C}[153,154]$. Simply averaging literature values (as in Table 1 in [152]) is misleading regarding $T_{\mathrm{S}}$ because non-equilibrium DSC indicates a transition temperature $T_{\mathrm{S}}$ in excess of $17^{\circ} \mathrm{C}$. Cooling scans also are misleading because nuclei of the subgel phase do not form readily near $T_{\mathrm{S}}$. To form the subgel phase requires lowering $T$ below $8^{\circ} \mathrm{C}$ for a few hours. Then, the subgel phase continues to grow even when $T$ is raised to any temperature below $T_{\mathrm{S}}$. It is also possible that there may be several different subgel phases with different structures, even for the same lipid, depending upon level of hydration and temperature. However, some of the many reported subgel phases are not equilibrium states, but are kinetic artifacts that occur when fast scanning rates are employed $[155,156]$. Thermal protocols for forming subgel phases are quite important to eliminate the effects of polycrystalline samples whose properties are corrupted by a large volume fraction of domain walls [155-157].

X-ray studies on DPPC $[158,159,161]$ and other phosphatidylcholines [162,163], as well as DPPG [160], show that there are reflections intermediate in $q$ between the low-angle (lamellar) and the wide-angle (chain packing) regions. We suggest that these intermediate angle reflections, absent in gel and ripple phases, should be the principal characteristic identifier of a subgel phase. Reflections in this region suggest order at the level of lipid molecules, although more crystalline chain packing also occurs and is another characteristic of subgel phases. The terminology $L_{\mathrm{c}}$ phase, where c denotes crystalline, is often used to describe this phase, but it should be emphasized that the wide angle diffraction patterns report order that propagates only within each bilayer and not to adjacent bilayers, so the subgel phase is not a three dimensional crystal. Within each bilayer the hydrocarbon chains are tilted between $30-35^{\circ}$ in DPPG [160] and the unit cell contains one molecule. In DPPC the chain tilt is $\theta_{\mathrm{t}}=34.5^{\circ}$ and the unit cell contains two lipid molecules [161]. Furthermore, Katsaras et al. [161] have advanced the likely interpretation that their DPPC data indicate ordering of the headgroups across the bilayer. This would contrast with just having an enlarged unit cell consisting of four crystalline hydrocarbon chains, although both pictures could involve molecular ordering within the same unit cell. However, there are still six possible motifs for headgroup ordering in DPPC that have yet to be resolved [161].

\section{Anomalous structure changes near $T_{M}$}

The effect of temperature is an illuminating probe of structure [13] and interactions [164]. We restrict this review of temperature studies to those dealing with a particularly interesting and controversial phenomenon, namely, the behavior of saturated chain lecithin bilayers as the main transition is approached from the fluid phase. The studies of Janiak et al. [13] already indicated that the coefficient of thermal expansion $\alpha=(\partial A / \partial T)$ increases anomalously near $T_{\mathrm{M}}$. Recent studies have focused on the accelerating increase in $D$ as $T$ is decreased towards $T_{\mathrm{M}}[80,165$ 171]. This increase is easily measured and all the data agree, but it is still controversial what causes the anomalous increase. Four models that involve critical phenomena near the main phase transition have been identified to explain the observed behavior [170]. These include (I) an unbinding transition $[80,166,167]$, (II) chain straightening [168], which is consistent with an area decrease [13], (III) changes in interbilayer interactions, and (IV) thickening of the interfacial region. Some experimental results obtain an increase in thickness of the water layer plus the interfacial headgroup region $[80,166,167,169,171]$, which have been interpreted in favor of model I 
[80,166,167,169] or model IV [171]. Other experimental results have found that half of the increase is due to bilayer thickening $[116,165,170]$. This suggests that the anomalous effect is due partly to model II but that at least one other model must also be involved [170]. If model II plays a role, then this phenomenon provides a sensitive way to tune bilayer thickness by adjusting thermodynamic parameters, $T$ being only the most obvious one, near the main transition within the $L_{\alpha}$ phase.

\section{Summary of structural results}

Table 6 summarizes our current best values for the parameters that characterize the structure of five $L_{\alpha}$ phase lipid bilayers and, for comparison, the gel phases of DPPC and DLPE. As was discussed in Section 3, the values of $V_{\mathrm{L}}$ and $D$ are quite accurate. The major issue is the best value of $A$, with various choices shown in Table 5 . It is likely that GX values are too high due to defect water. Our perspective is that the GXC values of $A$ still suffer from this problem, though to a much lesser extent than the GX values, and this is consistent with the overall comparison of GXC values of $A$ for DOPC and EPC with those determined by the EDP method; the latter re- sults for $A$ are therefore used in Table 6. For DMPC, we have chosen the EDP result which agrees with the GXC result of Koenig et al. [63]. For DPPC, the GXC result in Table 5 is anomalously larger than the EDP result and the EDP result is in closer agreement with the re-interpreted neutron diffraction and unilamellar results. For Table 6 we have chosen a compromise value between the EDP and neutron results. For DLPE the original result in [73] was used for the gel phase. The result for the $L_{\alpha}$ phase was corrected to $50.6 \AA^{2}$ by the authors [172] and slightly modified to $51.2 \AA^{2}$ due to improved volumetric data [26].

With the preceding values of $A$ and the measured $V_{\mathrm{L}}$ and $D$, the remaining quantities in Table 6 are obtained using the following calculations. The volume of the hydrocarbon chain region follows from $V_{\mathrm{C}}=V_{\mathrm{L}}-V_{\mathrm{H}}$ where $V_{\mathrm{H}}$ has been taken from gel phase studies to be $319 \AA^{2}$ for all lecithin headgroups in all phases. The average methylene volume $V_{\mathrm{CH}_{2}}$ is then obtained using the ratios of volumes of the terminal methyls and the double-bonded groups in the chains from Table 2 .

The reader may find it valuable, when considering the remaining quantities, to examine Fig. 2. That figure is drawn quantitatively accurate for $L_{\alpha}$ phase DPPC using the quantities in Table 6. The hydro-

Table 6

Final fully hydrated structural results ${ }^{\mathrm{a}}$

\begin{tabular}{|c|c|c|c|c|c|c|c|}
\hline Lipid & DPPC & DPPC & DMPC & DOPC & $\mathrm{EPC}$ & DLPE & DLPE \\
\hline Temperature & $20^{\circ} \mathrm{C}$ & $50^{\circ} \mathrm{C}$ & $30^{\circ} \mathrm{C}$ & $30^{\circ} \mathrm{C}$ & $30^{\circ} \mathrm{C}$ & $20^{\circ} \mathrm{C}$ & $35^{\circ} \mathrm{C}$ \\
\hline$V_{\mathrm{L}}\left(\AA^{3}\right)$ & 1144 & 1232 & 1101 & 1303 & 1261 & 863 & 907 \\
\hline $\mathrm{D}(\AA \stackrel{\mathrm{A}}{)})$ & 63.5 & 67 & 62.7 & 63.1 & 66.3 & 50.6 & 45.8 \\
\hline$A\left(\AA^{2}\right)$ & 47.9 & 64 & 59.6 & 72.5 & 69.4 & 41.0 & 51.2 \\
\hline$V_{\mathrm{C}}\left(\AA^{3} /\right.$ region $)$ & 825 & 913 & 782 & 984 & 942 & 611 & 655 \\
\hline$V_{\mathrm{CH}_{2}}\left(\AA^{3} /\right.$ group $)$ & 25.9 & 28.7 & 28.1 & 28.3 & - & 26.0 & 27.3 \\
\hline $2 D_{\mathrm{C}}(\AA)$ & 34.4 & 28.5 & 26.2 & 27.1 & 27.1 & 30.0 & 25.8 \\
\hline$D_{\mathrm{HH}}(\AA)^{\mathrm{b}}$ & 44.2 & 38.3 & 36.0 & 36.9 & 36.9 & 39.8 & 35.6 \\
\hline$D_{\mathrm{B}}^{\prime}(\AA)$ & 47.8 & 38.5 & 36.9 & 35.9 & 36.3 & 42.1 & 35.4 \\
\hline$D_{\mathrm{W}}(\AA)$ & 15.7 & 28.5 & 25.8 & 27.2 & 30.0 & 8.5 & 10.4 \\
\hline$D_{\mathrm{H}^{\prime}}(\AA)$ & 9.0 & 9.0 & 9.0 & 9.0 & 9.0 & 8.5 & 8.5 \\
\hline$D_{\mathrm{B}}^{\prime}(\AA)$ & 52.4 & 46.5 & 44.2 & 45.1 & 45.1 & 47.0 & 42.8 \\
\hline$D_{\mathrm{W}}^{\prime}(\AA)$ & 11.1 & 20.5 & 18.5 & 18.0 & 21.2 & 5.63 .6 & 5.03 .0 \\
\hline$n_{\mathrm{W}}$ & 12.6 & 30.1 & 25.6 & 32.8 & 34.7 & 5.8 & 8.8 \\
\hline$n_{\mathrm{W}}{ }^{\prime c}$ & 3.7 & 8.6 & 7.2 & 11.1 & 10.2 & 2.011 .0 & 4.73 .7 \\
\hline
\end{tabular}

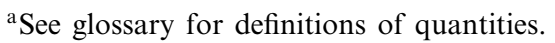

${ }^{\mathrm{b}}$ Using $D_{\mathrm{H} 1}=4.9$.

${ }^{\mathrm{c}}$ Calculated as $\left(A D_{\mathrm{H}}{ }^{\prime}-V_{\mathrm{H}}\right) / V_{\mathrm{W}}$. 
carbon thickness of the bilayer is given by $2 D_{\mathrm{C}}=2 V_{\mathrm{C}} / A$. In Fig. $2 \mathrm{c}$ the value of $D_{\mathrm{C}}$ compares favorably with the electron density profiles, the neutron diffraction distance for the $C_{4}$ methylene and the glycerol group, and the simulation distributions in Fig. 2a. The next entry in Table 6 is the head-head spacing $D_{\mathrm{HH}}$. Although this is the primary quantity determined by the electron density profiles, it is difficult to obtain precisely because the magnitude of the Fourier truncation corrections discussed in Section 5.1 depends upon the model employed to make them. However, the differences in $D_{\mathrm{HH}}$ used in Eq. 6 are relatively insensitive to the model, so the $A$ determination is more robustly determined than $D_{\mathrm{HH}}$. In Table 6 we have calculated $D_{\mathrm{HH}}$ for the phosphatidylcholine bilayers by adding $2 D_{\mathrm{C}}$ and $2 D_{\mathrm{H} 1}=9.8 \AA$; the latter comes from our most precise gel phase results and is only a little smaller than the value $2 D_{\mathrm{H} 1}=10.4 \AA$ given by the simulation shown in Fig. 2. A considerably smaller $2 D_{\mathrm{H} 1}=8.2 \AA$ was recently suggested [36], but we now believe that this value is too small. Simulations may continue to help to determine the value of $D_{\mathrm{H} 1}$.

In Table 6 the Gibbs-Luzzati bilayer thickness is $D_{\mathrm{B}}=2 V_{\mathrm{L}} / A$ and the corresponding water thickness is $D_{\mathrm{W}}=D-D_{\mathrm{B}}$. While these quantities are valuable for determining the effects of osmotic pressure (Eq. 2), they do not correspond well with the distributions of molecular components, as seen by comparing the left side of Fig. 2c with Fig. 2a. The steric thickness is calculated as $D_{\mathrm{B}}{ }^{\prime}=2 D_{\mathrm{C}}+2 D_{\mathrm{H}^{\prime}}{ }^{\prime}$. The assumed headgroup thicknesses, $D_{\mathrm{H}^{\prime}}=9 \AA$ for the PCs and $D_{\mathrm{H}}{ }^{\prime}=8.5 \AA$ for DLPE, are guided by neutron diffraction but are still somewhat arbitrary. McIntosh and Simon preferred $10 \AA$ [73] for the PCs and we once used $8 \AA$ [3]. $D_{\mathrm{H}^{\prime}}$ could be different for lecithin headgroups in different bilayers and phases due to differences in the phosphate-choline vector [36]. However, the correspondence between the electron density profiles and the simulation distributions in Fig. 2 suggests that $D_{\mathrm{H}^{\prime}}=9-10 \AA$ gives a reasonable measure of steric thickness. Then, the steric water thickness in Table 6 follows from $D_{\mathrm{W}}{ }^{\prime}=D-D_{\mathrm{B}}{ }^{\prime}$.

The stoichiometric amount of water $n_{\mathrm{W}}$ between bilayers in MLVs is calculated from Eq. 1. In Table 6 we also partition $n_{\mathrm{W}}$ into water that is in the steric water layer, located between $D_{\mathrm{B}}{ }^{\prime}$ and $D / 2$ in the transverse direction in Fig. $2 \mathrm{c}$ and into water that is in the interfacial region, located between $D_{\mathrm{C}}$ and $D_{\mathrm{B}}{ }^{\prime}$ in the transverse direction and between the headgroups in the lateral direction. We define the amount of the interfacial water as $n_{\mathrm{W}^{\prime}}$, which is simply calculated as $n_{\mathrm{W}}{ }^{\prime}=n_{\mathrm{W}}-\left(A D_{\mathrm{W}}{ }^{\prime} / V_{\mathrm{W}}\right)$. As one would expect, Table 6 shows that $n_{\mathrm{W}^{\prime}}$ is smallest for the gel phase, and becomes increasingly larger as $A$ increases so that more water must enter between the headgroups. One should, however, be careful not to overinterpret $n_{\mathrm{W}}$ ' as 'bound' or 'strongly interacting' water because some of the water located in the $D_{\mathrm{W}}$ ' layer that is not included in $n_{\mathrm{W}}{ }^{\prime}$ certainly interacts strongly with the headgroup, so $n_{\mathrm{W}}{ }^{\prime}$ might be considered as a lower estimate for bound water. This is consistent with measurements of unfreezable water, 5.5 for DMPC [173], 5 for DPPC [174] and 7 for DPPC [175], which indeed are somewhat larger than the value $n_{\mathrm{W}}{ }^{\prime}=3.7$ given for the gel phase of DPPC in Table 6. There is similar consistency between Table 6 and a recent NMR determination of 4.3 and 9.7 bound waters in the gel and $L_{\alpha}$ phases, respectively, of DMPC [176] and a recent radiolabel centrifugal determination of 8.6 in the $L_{\alpha}$ phase of DMPC [177].

As expected, Table 6 shows that the thickness $2 D_{\mathrm{C}}$ in the $L_{\alpha}$ phase, that would be used primarily for hydrophobic matching purposes, is smaller for the shorter DMPC chains than for the longer DPPC chains. However, the difference is not as great as one might expect. One naive way to predict these differences is to subtract a distance $D_{\mathrm{CH}_{2}}$ traversed by the extra methylenes; for fluid phase DPPC $D_{\mathrm{CH}_{2}}=0.90 \AA$ can be obtained from $2 V_{\mathrm{CH}_{2}} / A$. This would then predict that $2 D_{\mathrm{C}}$ for DMPC should be smaller than for DPPC by $3.6 \AA$, which disagrees with the difference of $2.4 \AA$ in Table 6 . The immediate reason is that DMPC has a smaller $A$ so that $D_{\mathrm{CH}_{2}}$ is slightly larger $(0.94 \AA)$ for each of its methylenes than for DPPC and the accumulated lengths of the chains can be quite different than the naive prediction. The deeper reason is that the temperature is $20^{\circ} \mathrm{C}$ higher for the DPPC study, and this causes the hydrocarbon chains to have more gauche rotamers and therefore to be shorter on a per methylene basis. Although the concept of reduced temperatures relative to the main transition has been invoked to favor the naive estimate above, this concept has no fundamental relevance for the first order 
main transition. Indeed, if one applies a thermal expansion adjustment for DMPC, one estimates $A_{\mathrm{DMPC}}^{50}=63.3 \AA^{2}$ at $50^{\circ} \mathrm{C}$, which is quite close to $A_{\mathrm{DPPC}}^{50}$. Finally, we note that values of $2 D_{\mathrm{C}}$ for DOPC and EPC are nearly the same, as expected since these are quite similar lipids with an average of one double bond on the $s n-2$ chain. Despite the larger numbers of carbons in their hydrocarbon chains, the double bond induces sufficient disorder to reduce $D_{\mathrm{C}}$ in DOPC and EPC nearly to that of DMPC, even though there is a difference of 4 carbons/chain. Similarly, despite having fewer carbons per chain, DLPE has nearly as large $D_{\mathrm{C}}$ as DMPC because its $A$ is so small.

\section{Conclusions and perspectives}

This review has shown that the great uncertainty in literature values for bilayer structure shown in Fig. 1 is being reduced by carefully considering adjustments and corrections to older literature values. We suggest giving more weight to a modern method that fully respects the liquid crystallinity of the fully hydrated, biologically most relevant, fluid $\left(L_{\alpha}\right)$ phase. The modern liquid crystallography method also sheds light on interbilayer interactions as discussed in Section 6. Our best estimates for the structural parameters for four phosphatidylcholines and one phosphatidylethanolamine are given in Table 6 . While we are fairly comfortable with these values, improvements in methodology should still be sought. Now that the vapor pressure paradox has been resolved, one methodological direction is the more systematic use of fully hydrated oriented samples to achieve higher spatial resolution. Another direction is the increased use of simulations to guide experimental analysis.

Of course, there are many other important lipids that should be characterized, both singly and in mixture, using the more rigorous methods reviewed here, in order to provide a compendium covering as many lipids as was given over ten years ago by Rand and Parsegian [1]. It is then expected that remaining issues concerning bilayer structure and interactions will be resolved and that this will provide a firm basis for further research on biomembranes.

\section{Acknowledgements}

We thank Klaus Gawrisch for supplying Fig. 3, Scott Feller for providing simulation data for Fig. 2, Evan Evans for discussion of Eq. 18 and providing current values of material moduli, Tom McIntosh for many discussions and Horia Petrache for improving the manuscript as well as for playing a key role in much of our research. This work was supported by grant GM44976 from the Institute of General Medical Sciences of the US National Institutes of Health.

\section{Appendix. Glossary of terms}

$A \quad$ average interfacial area/lipid (Fig. 2c, Eq. 1)

$v_{\mathrm{M}} \quad$ lipid-specific volume (Section 3.1)

$M_{\mathrm{L}} \quad$ molecular mass of lipid

$V_{\mathrm{L}} \quad$ lipid molecular volume, $V_{\mathrm{L}}=v_{\mathrm{M}} \times M_{\mathrm{L}} / .6023\left(\AA^{3}\right)$ (see Section 3.1)

$V_{\mathrm{W}} \quad$ water molecular volume, $\left(\approx 30 \AA^{3}\right.$, minor $\mathrm{T}$ dependence)

$V_{\mathrm{H}} \quad$ volume of head group, including glycerols and carbonyls (see Section 3.1)

$V_{\mathrm{C}} \quad$ sum of volumes of chain methylenes and methyls, $V_{\mathrm{C}}=V_{\mathrm{L}}-V_{\mathrm{H}}$, (see Section 3.1)

$V_{\mathrm{G}} \quad$ volume of component group $G$, e.g., $G=\mathrm{CH}_{2}$ (see Section 3.1)

$D \quad$ lamellar repeat spacing (see Fig. 2c and Section 3.2)

$D_{\mathrm{HH}} \quad$ headgroup peak-peak distance (Fig. $2 \mathrm{~b}$ and Section 5.1)

$D_{\mathrm{C}} \quad$ thickness of hydrocarbon core, $D_{\mathrm{C}}=V_{\mathrm{C}} / A$ (Fig. 2c, Section 2)

$D_{\mathrm{B}} \quad$ Gibbs-Luzzati bilayer thickness, $D_{\mathrm{B}}=2 V_{\mathrm{L}} / A$ (Fig. 2c, Section 2)

$D_{\mathrm{W}} \quad$ Gibbs-Luzzati water thickness, $D_{\mathrm{W}}=D-D_{\mathrm{B}}$ (see Section 2.)

$D_{\mathrm{B}}{ }^{\prime} \quad$ steric bilayer thickness, $D_{\mathrm{B}}{ }^{\prime}=2\left(D_{\mathrm{C}}+D_{\mathrm{H}}{ }^{\prime}\right)($ Fig. $2 \mathrm{c}$, Section 2)

$D_{\mathrm{W}^{\prime}} \quad$ steric water thickness, $D_{\mathrm{W}^{\prime}}=D-D_{\mathrm{B}}{ }^{\prime}$

$D_{\mathrm{H}}{ }^{\prime} \quad$ steric headgroup thickness, $D_{\mathrm{H}^{\prime}}=\left(D_{\mathrm{B}}{ }^{\prime} / 2\right)-D_{\mathrm{C}}$

$D_{\mathrm{H} 1} \quad$ partial headgroup thickness (Section 10.1) $D_{\mathrm{H} 1}=\left(D_{\mathrm{HH}} /\right.$ 2) $-D_{\mathrm{C}}$

$n_{\mathrm{W}} \quad$ number of water molecules/lipid, $n_{\mathrm{W}}=A D_{\mathrm{W}} / 2 V_{\mathrm{W}}$ (see Section 4.1)

$n_{\mathrm{W}}{ }^{\prime} \quad$ number of waters between $D_{\mathrm{C}}$ and $D_{\mathrm{B}}{ }^{\prime} / 2$ in Fig. 2c (see Section 12)

$q \quad$ scattering parameter, $4 \pi \sin \theta / \lambda$

$h \quad$ peak scattering order number, $q_{\mathrm{h}}=2 \pi h / D$

$F(q) \quad$ bilayer form factor (Eq. 4)

$S(q) \quad$ stacking structure factor (Eq. 9)

$K_{\mathrm{A}} \quad$ area compressibility modulus $(\mathrm{dyn} / \mathrm{cm})$ (Eq. 2, Section 4.2) 
$K_{\mathrm{C}} \quad$ bending modulus (ergs) (Section 5.4)

$B$ bulk compression modulus (erg/ $\mathrm{cm}^{4}$ ) (see Section 5.4)

$\alpha \quad$ area thermal expansivity, $(1 / A)(\partial A / \partial T)_{\pi}$

$\eta_{1} \quad$ Caillé parameter to quantitate fluctuations (see Section 5.4, Eq. 10)

EDP electron density profile (Section 5)

GX gravimetric X-ray method (Section 4.1)

GXC compressibility corrected gravimetric X-ray method (Section 4.2)

MLV multilamellar vesicle

VPP vapor pressure paradox (Section 3.2.2)

\section{References}

[1] R.P. Rand, V.A. Parsegian, Biochim. Biophys. Acta 988 (1989) 351-376.

[2] H.I. Petrache, K. Tu, J.F. Nagle, Biophys. J. 76 (1999) 2479 2487.

[3] J.F. Nagle, R. Zhang, S. Tristram-Nagle, W.-S. Sun, H.I. Petrache, R.M. Suter, Biophys. J. 70 (1996) 1419-1431.

[4] W.-J. Sun, R.M. Suter, M.A. Knewtson, C.R. Worthington, S. Tristram-Nagle, R. Zhang, J.F. Nagle, Phys. Rev. E 49 (1994) 4665-4676.

[5] F. Jähnig, Biophys. J. 36 (1981) 329-345.

[6] O.G. Mouritsen, M. Bloom, Biophys. J. 46 (1984) 293397.

[7] A. Killian, Biochim. Biophys. Acta 1376 (1998) 401-416.

[8] F. Dumas, M.C. Lebrun, J.F. Tocanne, FEBS Lett. 458 (1999) 271-277.

[9] R.J. Pace, S.I. Chan, J. Chem. Phys. 76 (1982) 4217-4227.

[10] G. Büldt, H.U. Gally, J. Seelig, G. Zaccai, J. Mol. Biol. 134 (1979) 673-691.

[11] H. Schindler, J. Seelig, Biochemistry 14 (1975) 2283-2287.

[12] B.A. Lewis, D.M. Engelman, J. Mol. Biol. 166 (1983) 211 217.

[13] M.J. Janiak, D.M. Small, G.G. Shipley, J. Biol. Chem. 254 (1979) 6068-6078.

[14] L.R. DeYoung, K.A. Dill, Biochemistry 27 (1988) 52815289.

[15] L.J. Lis, M. McAlister, N. Fuller, R.P. Rand, V.A. Parsegian, Biophys. J. 37 (1982) 657-666.

[16] R.L. Thurmond, S.W. Dodd, M.F. Brown, Biophys. J. 59 (1991) 108-113.

[17] W. Rawicz, K.C. Olbrich, T.J. McIntosh, D. Needham, E.A. Evans, Biophys. J. 79 (2000) 328-339.

[18] I. Pascher, M. Lundmark, P.-G. Nyholm, S. Sundell, Biochim. Biophys. Acta 1113 (1992) 339-373.

[19] M.C. Wiener, S.H. White, Biophys. J. 59 (1991) 162-173.

[20] H.I. Petrache, S.E. Feller, J.F. Nagle, Biophys. J. 72 (1997) 2237-2242.

[21] S.E. Feller, D. Yin, R.W. Pastor, A.D. MacKerrell, Biophys. J. 73 (1997) 2269-2279.

[22] R.S. Armen, O.D. Uitto, S.E. Feller, Biophys. J. 75 (1998) 734-744.
[23] K. Tu, D.J. Tobias, M.L. Klein, Biophys. J. 69 (1995) 25582562.

[24] J. Katsaras, V.A. Raghunathan, in: T. Gutberlet, J. Katsaras (Eds.), Lipid Bilayers: Structure and Interactions, Springer, New York, 2000.

[25] M.C. Wiener, S.H. White, Biophys. J. 61 (1992) 434447.

[26] J.F. Nagle, M.C. Wiener, Biochim. Biophys. Acta 942 (1988) $1-10$.

[27] V. Luzzati, F. Husson, J. Cell Biol. 12 (1962) 207-219.

[28] V.A. Parsegian, R.P. Rand, in: R. Lipowsky, E. Sackmann (Eds.), Structure and Dynamics of Membranes, North Holland, Amsterdam, 1995, pp. 643-690.

[29] J.F. Nagle, D.A. Wilkinson, Biophys. J. 23 (1978) 159175.

[30] M.C. Wiener, S. Tristram-Nagle, D.A. Wilkinson, L.E. Campbell, J.F. Nagle, Biochim. Biophys. Acta 938 (1988) 135-142.

[31] M. Posch, U. Rakusch, C. Mollay, P. Laggner, J. Biol. Chem. 258 (1983) 1761-1766.

[32] G. Schmidt, W. Knoll, Ber. Bunsenges. Phys. Chem. 89 (1985) 36-43.

[33] J.F. Blazyk, D.L. Melchior, J.M. Steim, Anal. Biochem. 68 (1975) 586-599.

[34] M. Bano, O. Pajdalova, Biophys. Chem. 80 (1999) 53-66.

[35] P. Laggner, H. Stabinger, in: M. Kerker (Ed.), Colloid and Interface Science, vol. V, Academic Press, New York, 1976, pp. 91-96.

[36] H.I. Petrache, S. Tristram-Nagle, J.F. Nagle, Chem. Phys. Lipids 95 (1998) 83-94.

[37] C.-H. Huang, J.P. Charlton, J. Biol. Chem. 246 (1971) 25552560.

[38] S. Tristram-Nagle, H.I. Petrache, J.F. Nagle, Biophys. J. 75 (1998) 917-925.

[39] M.C. Wiener, R.M. Suter, J.F. Nagle, Biophys. J. 53 (1989) 315-325.

[40] D.M. Small, in: The Physical Chemistry of Lipids: From Alkanes to Phospholipids, Plenum, New York, 1986, p. 494.

[41] S. Tristram-Nagle, R. Zhang, R.M. Suter, C.R. Worthington, W.-J. Sun, J.F. Nagle, Biophys. J. 64 (1993) 1097-1109.

[42] A. Tardieu, V. Luzzati, F.C. Reman, J. Mol. Biol. 75 (1973) 711-733.

[43] M.C. Wiener, S.H. White, Biochim. Biophys. Acta 938 (1988) 135-142.

[44] S.H. White, R.E. Jacobs, G.I. King, Biophys. J. 52 (1987) 663-665.

[45] R. Zhang, S. Tristram-Nagle, W.-J. Sun, R.L. Headrick, T.C. Irving, R.M. Suter, J.F. Nagle, Biophys. J. 70 (1996) 349-357.

[46] M.J. Janiak, D.M. Small, G.G. Shipley, Biochemistry 15 (1976) 4575-4580.

[47] H.I. Petrache, N. Gouliaev, S. Tristram-Nagle, R. Zhang, R.M. Suter, J.F. Nagle, Phys. Rev. E 57 (1998) 7014-7024.

[48] G.L. Jendrasiak, J.H. Hasty, Biochim. Biophys. Acta 337 (1974) 79-91.

[49] J. Torbet, W.H.F. Wilkins, J. Theor. Biol. 62 (1976) 447458. 
[50] F.Y. Chen, W.C. Hung, H.W. Huang, Phys. Rev. Lett. 79 (1997) 4026-4029.

[51] T.J. McIntosh, A.D. Magid, S.A. Simon, Biochemistry 26 (1987) 7325-7332.

[52] G.S. Smith, C. Safinya, D. Roux, N.A. Clark, Mol. Cryst. Liq. Cryst. 144 (1987) 235-255.

[53] J. Katsaras, D.S. Yang, R.M. Epand, Biophys. J. 63 (1992) $1170-1175$.

[54] S. Tristram-Nagle, H.I. Petrache, R.M. Suter, J.F. Nagle, Biophys. J. 74 (1998) 1421-1427.

[55] R. Podgornik, V.A. Parsegian, Biophys. J. 72 (1997) 942 952.

[56] J. Katsaras, Biophys. J. 75 (1998) 2157-2162.

[57] J. Katsaras, Biophys. J. 73 (1997) 2924-2929.

[58] J.F. Nagle, J. Katsaras, Phys. Rev. E 59 (1999) 7018 7024.

[59] D.M. LeNeveu, R.P. Rand, V.A. Parsegian, D. Gingell, Biophys. J. 18 (1977) 209-230.

[60] F. Reiss-Husson, J. Mol. Biol. 25 (1967) 363-382.

[61] S.M. Gruner, M.W. Tate, G.L. Kirk, P.T.C. So, D.C. Turner, D.T. Keane, C.P.S. Tilcock, P.R. Cullis, Biochemistry 27 (1988) 2853-2866.

[62] D.M. Small, J. Lipid Res. 8 (1967) 551-557.

[63] B.W. Koenig, H.H. Strey, K. Gawrisch, Biophys. J. 73 (1997) 1954-1966.

[64] T.J. McIntosh, A.D. Magid, S.A. Simon, Biochemistry 28 (1989) 7904-7912.

[65] K. Gawrisch, W. Richter, A. Mops, P. Balgavy, K. Arnold, G. Klose, Studia Biophys. 108 (1985) 5-16.

[66] T.J. McIntosh, S. Simon, Biochemistry 25 (1986) 4058-4066.

[67] G. Klose, B.W. Koenig, H.W. Meyer, G. Schulze, G. Degovics, Chem. Phys. Lipids 47 (1988) 225-234.

[68] M. Kodama, H. Aoki, H. Takahashi, I. Hatta, Biochim. Biophys. Acta 1329 (1997) 61-73.

[69] M.J. Ruocco, G.G. Shipley, Biochim. Biophys. Acta 691 (1982) 309-320.

[70] E.A. Evans, D. Needham, J. Phys. Chem. 91 (1987) 4219 4228.

[71] J.F. Nagle, M.C. Wiener, Biophys. J. 55 (1989) 309-313.

[72] W.-J. Sun, S. Tristram-Nagle, R.M. Suter, J.F. Nagle, Biophys. J. 71 (1996) 885-891.

[73] T.J. McIntosh, S.A. Simon, Biochemistry 25 (1986) 49484952.

[74] A. Caillé, C.R. Acad. Sci. Paris Série B 274 (1972) 891-893.

[75] J. Als-Nielsen, J.D. Litster, R.J. Birgeneau, M. Kaplan, C.R. Safinya, A. Lindegaad-Anderson, S. Mathiesen, Phys. Rev. B 22 (1980) 312-320.

[76] D. Roux, C.R. Safinya, J. Phys. France 49 (1988) 307-318.

[77] A. Guinier, in: X-Ray Diffraction, Freeman, San Francisco, 1963, pp. 300, 304.

[78] R. Hosemann, S.N. Bagchi, Direct Analysis of Diffraction by Matter, North-Holland, Amsterdam, 1962.

[79] S. Schwartz, J.E. Cain, E.A. Dratz, J.K. Blasie, Biophys. J. 15 (1975) 1201-1233.

[80] J. Lemmich, K. Mortensen, J.H. Ipsen, T. Honger, R. Bauer, O.G. Mouritsen, Phys. Rev. E 53 (1996) 5169-5180.
[81] T.J. McIntosh, S.A. Simon, Biochemistry 32 (1993) 8374 8384.

[82] R. Zhang, R.M. Suter, J.F. Nagle, Phys. Rev. E 50 (1994) 5047-5060.

[83] T.J. McIntosh, Curr. Opin. Struct. Biol. 10 (2000) 481-485.

[84] W. Helfrich, Z. Naturforsch. 33a (1978) 305-315.

[85] C.R. Safinya, E.B. Sirota, D. Roux, G.S. Smith, Phys. Rev. Lett. 62 (1989) 1134-1137.

[86] D. Sornette, N. Ostrowsky, J. Chem. Phys. 84 (1986) $4062-$ 4067.

[87] R. Podgornik, V.A. Parsegian, Langmuir 8 (1992) 557-562.

[88] T.J. McIntosh, S.A. Simon, Annu. Rev. Biophys. Biomol. Struct. 23 (1994) 27-51.

[89] S. Leikin, V.A. Parsegian, D.C. Rau, R.P. Rand, Annu. Rev. Phys. Chem. 44 (1993) 369-395.

[90] T.J. McIntosh, S. Advani, R.E. Burton, D.V. Zhelev, D. Needham, S.A. Simon, Biochemistry 34 (1995) 8520-8532.

[91] N. Gouliaev, J.F. Nagle, Phys. Rev. Lett. 81 (1998) 26102613.

[92] N. Gouliaev, J.F. Nagle, Phys. Rev. E 58 (1998) 881-888.

[93] N. Gouliaev, in: Interactions of Membrane Systems, Ph.D. Dissertation, Carnegie Mellon University, 1998.

[94] E.A. Evans, W. Rawicz, Phys. Rev. Lett. 64 (1990) 2094 2097.

[95] P. Méléard, C. Gerbeaud, T. Pott, L. Fernandez-Puente, I. Bivas, M.D. Mitov, J. Dufourcq, P. Bothorel, Biophys. J. 72 (1997) 2616-2619.

[96] V.A. Parsegian, Langmuir 11 (1995) 1047-1047.

[97] N. Lei, in: Synchrotron X-ray Scattering Studies of the Interactions and Undulations of Surfactant Fluid Multilayer Membranes, Ph.D. Dissertation, Rutgers University, 1993.

[98] F. Auguste, P. Barois, L. Fredon, B. Clin, D.J. Dufourc, A.M. Bellocq, J. Phys. II France 4 (1994) 2197-2214.

[99] R.J. Mashl, H.L. Scott, S. Subramaniam, E. Jakobsson, Biophys. J. (2000) submitted.

[100] K. Hristova, S.H. White, Biophys. J. 74 (1998) 2419-2433.

[101] L. Perera, U. Essman, M.L. Berkowitz, Prog. Colloid Polym. Sci. 103 (1997) 107-113.

[102] J.F. Nagle, Biophys. J. 64 (1993) 1476-1481.

[103] O. Berger, O. Edholm, F. Jähnig, Biophys. J. 72 (1997) 2002-2013.

[104] S.M. Smondyrev, M.L. Berkowitz, J. Chem. Phys. 110 (1999) 3981-3985.

[105] N.O. Petersen, S.I. Chan, Biochemistry 16 (1977) 26572667.

[106] A.A. Nevzorov, T.P. Trouard, M.F. Brown, Phys. Rev. E 58 (1998) 2259-2281.

[107] J.F. Faucon, M.D. Mitov, P. Meleard, I. Bivas, P. Bothorel, J. Phys. (France) 50 (1989) 2389-2414.

[108] H.I. Petrache, S.W. Dodd, M.F. Brown, Biophys. J. (2000) accepted.

[109] G. Niggemann, M. Kummrow, W. Helfrich, J. Phys. II France 5 (1995) 413-425.

[110] K.H. de Haas, C. Blom, D. van den Ende, M.H.G. Duits, J. Mellema, Phys. Rev. E 56 (1997) 7132-7137. 
[111] H.P. Duwe, J. Kaes, E. Sackmann, J. Phys. France 50 (1990) 945-962.

[112] J.F. Nagle, Y. Liu, S. Tristram-Nagle, R.M. Epand, R.W. Stark, Biophys. J. 77 (1999) 2062-2066.

[113] Z. Zhou, B.G. Sayer, D.W. Hughes, R.E. Stark, R.M. Epand, Biophys. J. 76 (1999) 387-399.

[114] P. Balgavy, M. Dubnickova, D. Uhrikova, S. Yaradaikin, M. Kiselev, V. Gordeliy, Acta Phys. Slovaca 48 (1998) 509 533.

[115] P.C. Mason, B.D. Gaulin, R.M. Epand, G.D. Wignall, J.S. Lin, Phys. Rev. E 59 (1999) 3361-3367.

[116] P.C. Mason, B.D. Gaulin, R.M. Epand, J. Katsaras, Phys. Rev. E 61 (2000) 5634-5639.

[117] C.W. Meuse, S. Krueger, C.F. Majkrzak, J.A. Dura, J. Fu, J.T. Connor, A.L. Plant, Biophys. J. 74 (1998) 1388-1398.

[118] H. Brockman, Curr. Opin. Struct. Biol. 9 (1999) 438-443.

[119] D. Marsh, Biochim. Biophys. Acta 1286 (1996) 183-223.

[120] J.F. Nagle, Faraday Discuss. Chem. Soc. 81 (1986) 151162.

[121] F. Jähnig, Biophys. J. 46 (1984) 687-694.

[122] S. Feng, Langmuir 15 (1999) 998-1010.

[123] J.M. Crane, G. Putz, S.B. Hall, Biophys. J. 77 (1999) 3134 3143.

[124] D.W.R. Gruen, J. Phys. Chem. 89 (1985) 153-163.

[125] A. Georgallis, D.A. Pink, Can. J. Phys. 60 (1982) 1678 1681.

[126] D. Vaknin, K. Kjaer, J. Als-Nielsen, M. Losche, Biophys. J. 59 (1991) 1325-1332.

[127] J. Katsaras, J. Phys. Chem. 99 (1995) 4141-4147.

[128] J.F. Nagle, Biophys. J. 64 (1993) 1110-1112.

[129] E.N. Serrallach, G.A. deHaas, G.G. Shipley, Biochemistry 23 (1984) 713-720.

[130] S. Tristram-Nagle, Y. Isaacson, Y. Lyatskaya, Y. Liu, K. Brummond, J. Katsaras, J.F. Nagle, Chem. Phys. Lipids 100 (1999) 101-113.

[131] R.M. Venable, B.R. Brooks, R.W. Pastor, J. Chem. Phys. 112 (2000) 4822-4832.

[132] J.F. Nagle, J. Membr. Biol. 27 (1976) 233-250.

[133] T.J. McIntosh, Biophys. J. 29 (1980) 237-246.

[134] W. Sun, S. Tristram-Nagle, R.M. Suter, J.F. Nagle, Biochim. Biophys. Acta 1279 (1996) 2094-2097.

[135] R.G. Snyder, G.L. Liang, H.L. Strauss, R. Mendelsohn, Biophys. J. 71 (1996) 3186-3198.

[136] T.J. McIntosh, S.A. Simon, J.C. Ellington, N.A. Porter, Biochemistry 23 (1984) 4038-4044.

[137] R.V. McDaniel, T.J. McIntosh, S.A. Simon, Biochim. Biophys. Acta 731 (1983) 97-108.

[138] C.-H. Huang, S. Li, Biochim. Biophys. Acta 1422 (1999) 273-307.

[139] R.N.A.H. Lewis, R. McElhaney, Biophys. J. 65 (1993) 1866-1877.

[140] T. Zhu, M. Caffrey, Biophys. J. 65 (1993) 939-954.

[141] K.M.W. Keough, P.J. Davis, Biochemistry 18 (1979) 1453 1459.

[142] K. Lohner, A. Schuster, G. Degovics, K. Muller, P. Laggner, Chem. Phys. Lipids 44 (1987) 61-70.
[143] K. Saxena, P. Zimmerman, R.R. Schmidt, G.G. Shipley, Biophys. J. 78 (2000) 306-312.

[144] J. Katsaras, S. Tristram-Nagle, Y. Liu, R.L. Headrick, E Fontes, P.C. Mason, J.F. Nagle, Phys. Rev. E 61 (2000) 5668-5677.

[145] M.P. Hentschel, F. Rustichelli, Phys. Rev. Lett. 66 (1991) 903-906.

[146] D.C. Wack, W.W. Webb, Phys. Rev. A 40 (1989) 27122730.

[147] W. Sun, S. Tristram-Nagle, R.M. Suter, J.F. Nagle, Proc. Natl. Acad. Sci. USA 93 (1996) 7008-7012.

[148] K. Sengupta, V.A. Raghunathan, J. Katsaras, Europhys. Lett. 49 (2000) 722-728.

[149] H. Yao, S. Matuoka, B. Tenchov, I. Hatta, Biophys. J. 59 (1991) 252-255.

[150] S. Matuoka, H. Yao, S. Kato, I. Hatta, Biophys. J. 64 (1993) 1456-1460.

[151] J.T. Woodward, J.A. Zasadzinski, Biophys. J. 72 (1997) 964-976.

[152] R. Koynova, M. Caffrey, Biochim. Biophys. Acta 1376 (1998) 91-145.

[153] J.F. Nagle, D.A. Wilkinson, Biochemistry 16 (1982) 38173821.

[154] M. Kodama, H. Hashigami, S. Seki, Biochim. Biophys. Acta 814 (1985) 300-306.

[155] S. Tristram-Nagle, M. Wiener, C.-P. Yang, J.F. Nagle, Biochemistry 26 (1987) 4288-4294.

[156] S. Tristram-Nagle, R.M. Suter, W.-J. Sun, J.F. Nagle, Biochim. Biophys. Acta 1191 (1994) 14-20.

[157] C.P. Yang, J.F. Nagle, Phys. Rev. A 37 (1988) 3993-4000.

[158] M.J. Ruocco, G.G. Shipley, Biochim. Biophys. Acta 684 (1982) 59-66.

[159] M.J. Ruocco, G.G. Shipley, Biochim. Biophys. Acta 691 (1982) 309-320.

[160] A.E. Blaurock, T.J. McIntosh, Biochemistry 25 (1986) 299 305 .

[161] J. Katsaras, V.A. Raghunathan, E.J. Dufourc, J. Dufourcq, Biochemistry 34 (1995) 4686-4688.

[162] J. Stumpel, H. Eibl, A. Nicksch, Biochim. Biophys. Acta 727 (1983) 246-254.

[163] V.A. Raghunatahan, J. Katsaras, Phys. Rev. E 54 (1996) 4446-4449.

[164] S.A. Simon, S. Advani, T.J. McIntosh, Biophys. J. 69 (1995) 1473-1483.

[165] S. Kirchner, G. Cevc, Europhys. Lett. 23 (1993) 229-235.

[166] T. Honger, K. Mortensen, J.H. Ipsen, J. Lemmich, R. Bauer, O.G. Mouritsen, Phys. Rev. Lett. 72 (1994) 39113914.

[167] J. Lemmich, K. Mortensen, J.H. Ipsen, T. Honger, R. Bauer, O.G. Mouritsen, Phys. Rev. Lett. 75 (1995) 39583961.

[168] R. Zhang, S. Tristram-Nagle, R.L. Headrick, R.M. Suter, J.F. Nagle, Phys. Rev. Lett. 74 (1995) 2832-2835.

[169] F.Y. Chen, W.C. Hung, H.W. Huang, Phys. Rev. Lett. 79 (1997) 4026-4029.

[170] J.F. Nagle, H.I. Petrache, N. Gouliaev, S. Tristram-Nagle, 
Y. Liu, R.M. Suter, K. Gawrisch, Phys. Rev. E 58 (1998) 7769-7776.

[171] F. Richter, L. Finegold, G. Rapp, Phys. Rev. E 59 (1999) 3483-3492.

[172] T.J. McIntosh, S.A. Simon, Biochemistry 25 (1986) 49484952.

[173] H. Aoki, M. Kodama, Thermochim. Acta 308 (1987) 7783.

[174] V.L. Bronshteyn, P.L. Steponkus, Biophys. J. 65 (1993) $1853-1865$.
[175] L.M. Crowe, B.J. Spargo, T. Ioneda, B.L. Beaman, J.H. Crowe, Biochim. Biophys. Acta 1194 (1994) 53-60.

[176] C. Faure, L. Bonakdar, E.J. Dufourc, FEBS Lett. 405 (1997) 263-266.

[177] S. Channareddy, S.S. Jose, N. Janes, J. Am. Chem. Soc. 119 (1997) 2345-2347.

[178] N. Lei, X. Lei, Langmuir 14 (1998) 2155-2159.

[179] E. Lindahl, O. Edholm, Biophys. J. 79 (2000) 426-433.

[180] S.E. Feller, Y. Zhang, R.W. Pastor, J. Chem. Phys. 103 (1995) 10267-10276. 